\title{
Financial Risk Early Warning Based on Wireless Network Communication and the Optimal Fuzzy SVM Artificial Intelligence Model
}

\author{
Yong Ma, ${ }^{1}$ Hao Liu, ${ }^{1}$ Guangyu Zhai ${ }^{(}{ }^{2}$ and Zongjie Huo ${ }^{2}$ \\ ${ }^{1}$ State Grid Gansu Electric Power Company, Lanzhou, 730030 Gansu, China \\ ${ }^{2}$ School of Economics and Management, Lanzhou University of Technology, Lanzhou, 730050 Gansu, China \\ Correspondence should be addressed to Guangyu Zhai; zhaigy@lzu.edu.cn
}

Received 6 August 2021; Revised 11 November 2021; Accepted 24 November 2021; Published 23 December 2021

Academic Editor: Zhihan Lv

Copyright (c) 2021 Yong Ma et al. This is an open access article distributed under the Creative Commons Attribution License, which permits unrestricted use, distribution, and reproduction in any medium, provided the original work is properly cited.

\begin{abstract}
Since the beginning of the new century, risk events such as the world economic crisis have occurred, which have greatly impacted the real economy of our country. A wireless network is a network implemented using wireless communication technology. It includes both global voice and data networks that allow users to establish long-distance wireless connections, as well as infrared technology and radio frequency technology optimized for short-distance wireless connections. These events have a great impact on many small- and medium-sized listed companies, resulting to many small- and medium-sized listed companies going bankrupt. Indeed, one of the important reasons for the frequent bankruptcy of small- and medium-sized listed companies is the lack of awareness of risk prevention and effective financial risk early warning mechanism. The support vector machine is a machine learning method based on the VC dimension theory of statistical learning and the principle of structural risk minimization. This method shows many unique advantages when dealing with classification problems and has been widely used in many fields. The purpose of this article is to realize the financial risk analysis of listed companies through wireless network communication and the optimal fuzzy SVM artificial intelligence model, which help small- and medium-sized listed companies find abnormalities in their business management activities in advance and deal with market risks in a timely manner. Taking 81 small- and medium-sized listed companies as the research object, this paper chooses the small- and medium-sized listed companies in every quarter of 2018 as the research sample. By using the financial and nonfinancial data of small- and medium-sized listed companies and introducing the support vector machine (SVM) with the fuzzy method, the model of the fuzzy support vector machine (FSVM) is constructed. And the performance of the FSVM under four different kernel functions is compared and studied. At the same time, the performance of the FSVM is compared with other artificial intelligence models. The empirical results show that different kernel functions have different effects on the prediction performance of the FCM-SVM model. Under the Gauss radial basis function, the prediction accuracy of the FCM-SVM is over $86 \%$. It can be seen that in predicting the financial crisis of small- and medium-sized listed companies, the FCM-SVM model with Gauss radial basis function has the best predictive performance. The FSVM model based on Gauss radial basis function not only has the advantages of linearity, being polynomial, and nonlinearity of neurons but also is significantly superior to the traditional artificial intelligence model.
\end{abstract}

\section{Introduction}

In recent years, the state has vigorously advocated inclusive finance and established financing guarantee companies for small- and medium-sized listed companies to support and encourage the development of small- and medium-sized listed companies. Under the policy support environment, the development momentum of small- and medium-sized listed companies in China is rapid. At the same time, China's small- and medium-sized listed companies have a series of problems, such as fierce market competition, inadequate financial risk management capabilities, and imperfect corporate governance structure. In recent years, the occurrence of risk events such as the international financial crisis has 
greatly impacted the real economy of our country and led to the bankruptcy of a large number of small- and mediumsized listed companies [1, 2]. Fuzzy support vectors are supervised learning models and related learning algorithms that use classification and regression analysis to analyze data. By adjusting the weights of these coefficients, the artificial intelligence model allows data scientists to be trained with different results. The core requirement for the success of the algorithm is to have clear data without too much noise (low-value information) and delete input variables with similar values (related input values), for example, banking, insurance, medical, marketing, and other industries that can optimize financial statistics. Indeed, the lack of awareness of risk prevention and effective financial crisis early warning mechanism is one of the important reasons for the frequent bankruptcy of small- and medium-sized listed companies. The formation of financial crisis of small- and medium-sized listed companies is affected not only by financial factors but also by the lack of managers' professional ability and the imperfect corporate governance mechanism. In the postfinancial crisis era, the market operation risk is still significant. How can small- and medium-sized listed companies withstand risks and prevent risks in the complex living environment has become the focus of academic and practical circles. Nowadays, the application of wireless network communication is increasing rapidly. In academia, medical industry, manufacturing, warehousing, etc., wireless network communication is playing an increasingly important role in all walks of life. Through it, our lives become more intelligent and convenient.

With the acceleration of the process of economic globalization, the competition of small- and medium-sized listed companies is becoming increasingly fierce, which makes the possibility of financial crisis outbreak being greatly increased [3]. Once the financial crisis breaks out, it not only threatens the survival and development of small- and medium-sized listed companies but also seriously damages the investment interests of investors and even brings severe challenges to the development of the national economy [4]. Therefore, it is of great practical significance to carry out early warning research on financial crisis to accurately predict and effectively prevent and resolve financial crisis for promoting the healthy development of small- and medium-sized listed companies, optimizing the investment decisions of investors and promoting the stable development of the national economy, especially for the subprime mortgage crisis, European debt crisis, and other financial crises. Following the outbreak, financial crisis, early warning research has become one of the hot issues in the theoretical and practical circles $[5,6]$. Therefore, it is of great practical significance to study the early warning of the financial crisis of small- and medium-sized listed companies in the economic zone to maintain the stable operation and development of small- and medium-sized listed companies and promote the prosperity and harmony of the economic zone.

The kernels of SVM mainly include the linear kernel, polynomial kernel, Gauss radial basis function kernels, and the nonlinear interaction kernels of neurons [7, 8]. However, there is no unified conclusion about which kind of kernel function can make the SVM early warning model achieve the best prediction effect. Although some scholars have proved through empirical research that the prediction effect of the SVM model based on Gauss radial basis function is the best, due to the inconsistency of the research object, the SVM model based on Gauss radial basis function may not achieve the optimal prediction effect $[9,10]$ in the financial crisis early warning of small- and medium-sized enterprises in the economic zone. Based on this, this paper will build different FSVM models based on the above four kinds of kernels and explore an optimal FSVM model, to provide an effective decision-making reference for small- and medium-sized enterprises in the economic zone to control and prevent financial crisis [11, 12]. Based on the above analysis, this paper takes small- and medium-sized enterprises in an economic zone as the research object, constructs different FSVM financial crisis early warning models based on four different kernel functions, and compares and studies a kind of FSVM early warning model with the best predictive performance, to play an important role in promoting the healthy operation of small- and medium-sized enterprises in the economic zone and the stable development of the economy in the economic zone.

The structure of this paper is as follows: the first part introduces the background and significance of this study, as well as the innovation and organization of this paper; the second part introduces the related research of predecessors in this field, machine learning, and other related theories, as well as the model of the support vector machine. The third part introduces the data source, data sample partition, and LIBSVM toolbox of the experiment. The fourth part discusses the following: first, the comparative analysis of different kernels in the experiment, second, the comparative analysis of the performance of different early warning models, third, the McNemar test result analysis of different early warning models, fourth, the logistic model analysis, and fifth, the discussion of the causes of financial crisis. The fifth part summarizes the related research work and draws relevant conclusions.

\section{Proposed Method}

2.1. Related Work. Hang proposed a CCPR algorithm which combines the fuzzy support vector machine (SVM) with hybrid kernel function and genetic algorithms (GA). Firstly, two shape features and two statistical features independent of distribution parameters and sample number are proposed to describe the characteristics of cops. Then, a multiclass classification method based on the hybrid kernel function fuzzy support vector machine is proposed. By assigning a certain degree of membership to each training sample, this method weakens the influence of outliers on the classification accuracy of classifiers based on the support vector machine. At the same time, a hybrid kernel function combining Gauss kernel and polynomial kernel is used to further improve the generalization ability of the classifier. To solve the problem of feature selection and parameter optimization, a genetic algorithm is used to optimize the input feature subset and parameters of the classifier based on the fuzzy 
support vector machine. Finally, the feasibility and effectiveness of the method are verified by simulation experiments and examples. The simulation results show that this method can achieve better CCPR performance than other methods, such as the learning vector quantization network, multilayer perceptron network, probabilistic neural network, fuzzy clustering, and support vector machine. In recognition accuracy, the example shows that this method has potential application to solve the interpretation problem of the actual control charts. However, in their experimental research, there are still some problems in the experiment and all influencing factors have not been eliminated technically. The result is not so perfect $[13,14]$.

The support vector machine (SVM) is a machine learning method that can complete classification tasks. Many researchers use support vector machine libraries to accelerate their research and development [15, 16]. Using such libraries saves time and avoids writing the code from scratch. LIBSVM is one of the support vector machine libraries, which is widely used by researchers to solve problems. The library also integrates Weka, a popular data mining tool. This paper includes our work on complexity analysis of support vector machines. Abdiansah and Wardoyo study the support vector machine algorithm and its implementation in LIBSVM. Abdiansah and Wardoyo also use two popular programming languages, $\mathrm{C}++$ and Java, to test our analysis and experiments using three different datasets. The results show that the complexity of the support vector machine (LIBSVM) is o. The time complexity shows that $\mathrm{C}++$ is faster than Java in training and testing, and the increase of data will affect and increase the computing time $[17,18]$.

PoliszcWei et al. used the fuzzy support vector machine algorithm to select AGN candidates, which allows the introduction of measurement uncertainty in the classification process. The training dataset is based on spectral data that can be used to select objects in the NEP deep field and NEP wide field. The synthetic samples are based on AKARI NEP-Deep Field data, including objects without optical counterparts, using only infrared information. A highquality catalogue of 275 AGN candidates not previously classified was compiled [19, 20].

Thaseen and Kumar presented an intrusion detection model based on chi-square feature selection and the multiclass support vector machine (SVM). The parameter tuning technique is used to optimize the core parameter gamma of the radial basis function. Gamma is expressed as an overfitting constant. These are two important parameters for the support vector machine model [21]. The main idea of this model is to construct a multiclass support vector machine which has not been used for IDS so far, to reduce the training and testing time of network attacks and improve the accuracy of individual classification of network attacks. The experimental results on the improved NSL-KDD dataset of KDDCUP 1999 show that the proposed method has a better detection rate and lower false alarm rate. The computational time required for training and testing is also experimented for time-critical applications [22, 23].
2.2. Wireless Network Communication. The so-called wireless network refers to a network that can realize the interconnection of various communication devices without wiring. Wireless network technology covers a wide range, including global voice and data networks that allow users to establish long-distance wireless connections, as well as infrared and radio frequency technologies optimized for short-range wireless connections. According to the different network coverage, wireless networks can be divided into a wireless wide area network (WWAN), wireless local area network (WLAN), wireless metropolitan area network (WMAN), and wireless personal local area network (WPAN), as shown in Figure 1.

\subsection{Correlation Theory}

2.3.1. Machine Learning Theory. In human wisdom, the ability to learn and promote is an important ability. First of all, people can learn, analyze, and summarize from things, extract experience, and acquire laws and knowledge to continue to inherit and improve. In this process, people can not only explain the known things better but also predict the results that cannot be observed directly and make correct judgments and predictions for the future. Machine learning (ML) is a scientific research in which computer systems do not use explicit instructions to perform specific tasks effectively but rely on algorithms and statistical models used for patterns and inference. It is regarded as a subset of artificial intelligence. Machine learning algorithms build a mathematical model based on sample data, called "training data," to make predictions or decisions without explicit programming to perform tasks. Machine learning algorithms are used in various applications, such as email filtering and computer vision. In these applications, it is not feasible to develop algorithms for specific instructions to perform tasks. Machine learning is closely related to computational statistics, which focuses on the use of computers to make predictions. The research of algorithm optimization provides methods, theories, and application areas for the field of machine learning. Data mining is a research field in machine learning, focusing on exploratory data analysis for unsupervised learning. In the application of crossbusiness problems, machine learning is also called predictive analysis. The research of machine learning is expected to simulate the ability of learning and promotion. To realize these two abilities, pattern recognition and integrated learning are indispensable. The machine learning equipment is shown in Figure 2.

2.3.2. Pattern Recognition. Patterns refer to things that can be observed and analyzed. Whether in terms of time, space, or data, the premise of patterns is that they can be distinguished, that is, they can get the information that they can analyze through observations and determine the category that they belong to. In other words, patterns mean that time, space, and data can be observed and analyzed. The premise of the models is that they can be distinguished. They can be observed and classified by analyzing their information. Pattern recognition specifically refers to the processing and 


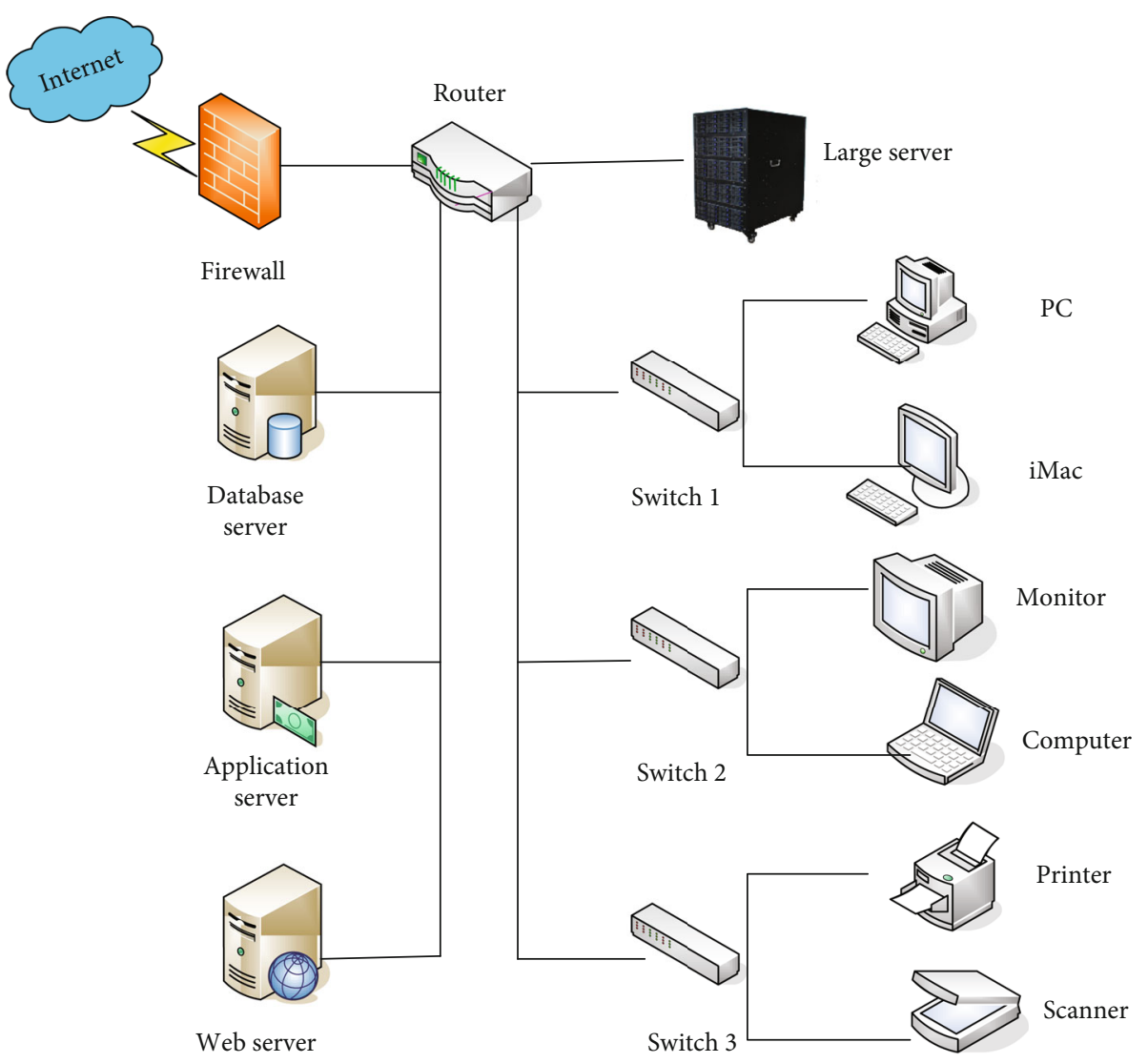

FIGURE 1: Wireless network communication.
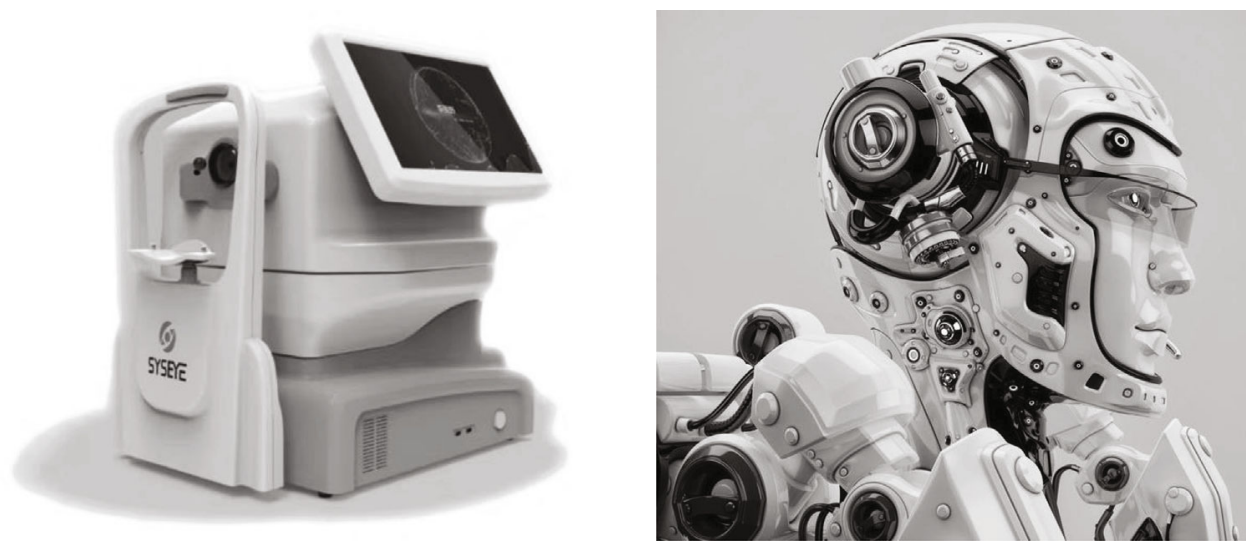

FIGURE 2: Machine learning equipment diagram.

analysis of various information forms, such as things and phenomena and, in this process, can identify, judge, classify, and analyze them. In other words, pattern recognition is the process of categorizing specific objects into specific categories. Even if a certain number of samples are used, classifiers are designed according to their differences, and then, classifiers are designed to categorize the identified samples. The purpose of pattern recognition research is to analyze, describe, judge, and classify all kinds of things and phenomena by computer and to make the recognition results consistent with the target object under the condition of minimizing the error probability. As shown in Figure 3.
Human beings are engaged in and complete pattern recognition activities in every link of life or learning. First of all, people can learn, analyze, and summarize from things, extract experience, and acquire laws and knowledge to continue to inherit and improve. In this process, people can not only explain well what is known but also predict accurately what cannot be observed directly and make correct judgments and predictions for the future. However, it is very difficult for computers. The most basic way to identify things is to calculate the similarity between the things that need computer analysis and standard templates. The exact difference between things to be determined is the difference between 


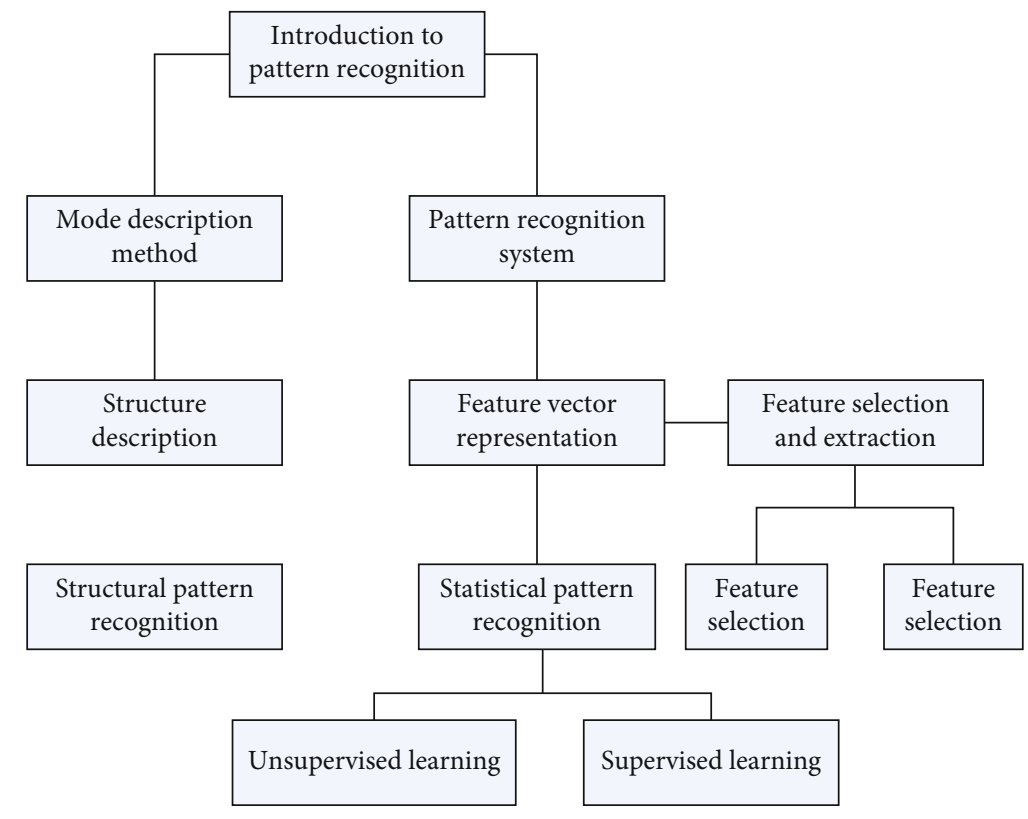

Figure 3: Pattern recognition structure.

different things that can be seen from the measures. Therefore, the key to pattern recognition is to find a way to measure the difference between different categories of things. The whole pattern recognition process is divided into three stages: data conversion stage, analysis stage, and classification stage. Data conversion is the most basic stage, which will not be discussed here. The second stage is to analyze the data after processing the result transformation, including the process of feature selection, feature extraction, and simple attributes. Classification is based on the analysis of information acquisition, using a classification algorithm to train and ultimately to get the classification rules with strong generalization ability. The three stages of the process can be summarized into two modes: training stage and testing stage.

2.3.3. Ensemble Learning. Learning and promoting ability requires the human brain and sensory system to undergo a complete process of activity-the brain completes a series of complex processes of analysis, judgment, and decisionmaking by processing the information received by the senses. However, without decomposing the whole process, it is difficult to achieve the human brain in any way. In addition to the treatment of neural network models, people also get inspiration from the macrointegration of group division of labor and cooperation in nature and explore machine learning methods to solve the same problem using a variety of learning machines. Different from the traditional single learning machine, ensemble learning uses multiple learning machine training based on different assumptions and then combines the training results to output the final results. Ensemble learning can be divided into two categories: one is to construct a base classifier with fixed process and combined weighted voting and the other is to construct a random classifier with random process and without combined weighted voting. The principle of integrated learning is shown in Figure 4.
Integrated learning has good generalization performance and stability. The difference of different learning modes can be used to improve the learning ability. The difference between the accuracy of the single learning model and the learning model is the main factor affecting the learning effect. The method of ensemble learning can be divided into two aspects: the construction of classifiers and the combination of classifiers. At present, scholars generally believe that if the errors generated by different sublearning are independent of each other and show diversity, the integrated learning system will perform better. Many scholars have studied this point. Unfortunately, research has shown that generating sublearners independently of each other is not an easy task. Firstly, for the same problem, we construct a group of different sublearners, which can be different parameters of a sublearner or different types of sublearners. Then, we synthesize the predicted results of each sublearner according to certain strategies. We know that it is very difficult to obtain high-precision learning in machine learning. Fortunately, integrated learning integrates individual accuracy. As long as the result is slightly better than the random guess, general learning can satisfy this condition. Therefore, the integrated learning algorithm mainly considers how to make the differences between sublearners effective. Achieving better integration effects has become the research focus of ensemble learning.

2.3.4. Statistical Learning Theory. Statistical learning theory starts from some observation (training) samples, trying to obtain some laws that cannot be analyzed in principle, and uses these laws to analyze objective objects, so that the laws can be used to make more accurate predictions on future data. For example, to predict the population of the country in the next few years, it is necessary to collect the population data of the past few years or even decades and make a statistical analysis and summary of its changing laws, to obtain an 


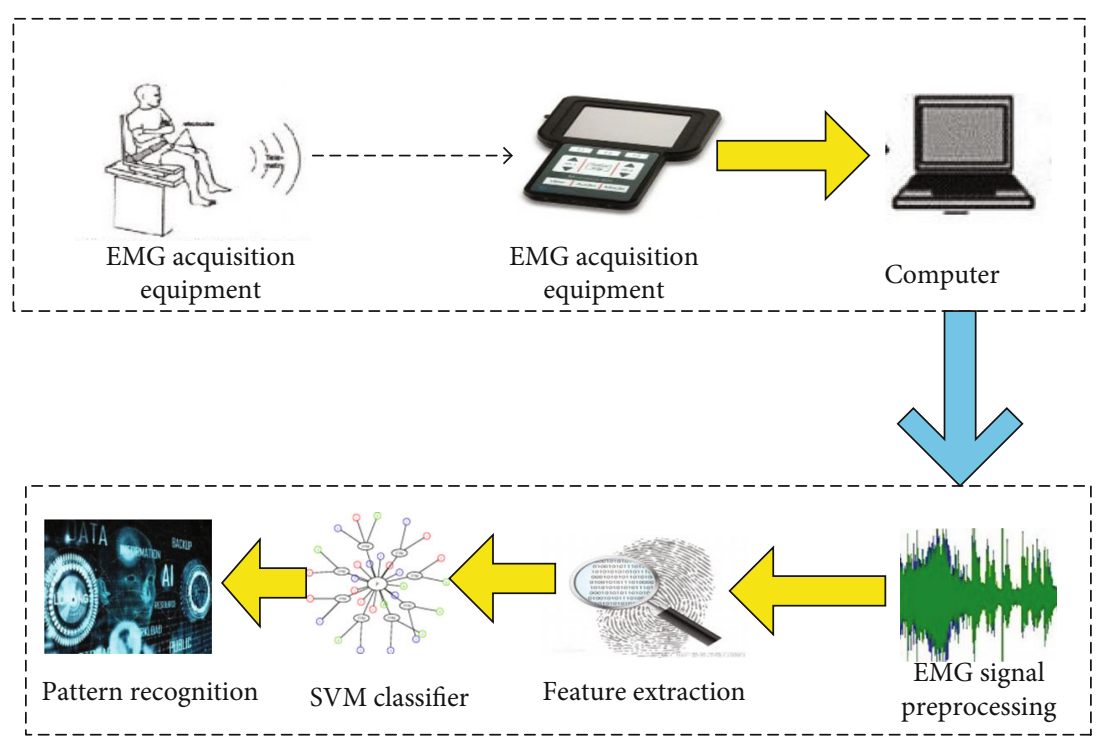

Figure 4: Principles of integrated learning.

overall prediction model, so that you can make a rough estimate and forecast of the overall population trend in the next few years. Statistical learning theory is essential and is the common basic theory of machine learning methods. Compared with the traditional machine, statistical machine learning can accurately give the learning effect and can answer a series of questions such as the number of samples needed.

\subsection{SVM Model}

2.4.1. Support Vector Machine. Support vector machine (SVM), also known as support vector network, is a supervised learning model and related learning algorithms that use classification and regression analysis to analyze data. Given a set of training samples, each training sample is marked as belonging to one or the other of the two categories. The support vector machine (SVM) training algorithm creates a model that assigns a new sample to one of the two categories, making it a nonprobabilistic binary linear classifier (although in the probabilistic classification setting, there is a model like Prato correction and such methods use support vector machines). The support vector machine model represents samples as mapped points in space, so that samples of a single category can be separated as clearly as possible. All such new samples are mapped to the same space, and it is possible to predict which category they belong to based on which side of the interval they fall on. At present, SVM is widely used in classification and regression. It regards the optimization training error as the optimization purpose, and the ultimate goal is to minimize the value of the confidence range. Analyzing the SVM algorithm, we can find three advantages: first, the SVM avoids the problem of local extremum. For example, neural network (BP) algorithm has this difficult problem. Secondly, when searching for the optimal classification solution, the SVM algorithm searches for the optimal classification solution based on the limited number of sample data, which is not the best result when search- ing for infinite data information. $H$, its generalization ability, and application ability on scientific issues are higher than some traditional methods, and the complexity of its calculation is not directly related to the ingenious data dimension. Therefore, SVM has significant advantages over other methods in dealing with high-dimensional pattern recognition, nonlinear problems, and small sample problems. The core idea of the SVM classifier is to use mathematical methods to establish the most appropriate classification boundaries and eventually classify the two sample data that need to be separated.

The optimal classification surface of the SVM is developed in the linear separable case. Its basic idea can be explained by the case of binary classification. As shown in Figure 5, the hollow point and solid point represent two different types of samples, namely, the classification line, the nearest sample passing through each kind of mid-off classification line, and the straight line parallel to the classification line. The shortest distance from the hyperplane to the hyperplane is called the classification interval. The optimal classification surface requires that the classification hyperplane ensures the correct segmentation of samples and maximizes the classification interval.

The equation for classifying hyperplanes is that $w$ is the normal value of the hyperplane, the Euclidean norm, and the vertical distance from the hyperplane to the origin. The classification hyperplane makes the linear separable sample set satisfy the following:

$$
\begin{array}{ll}
x_{i} w+b \geq+1, & \text { for } y_{i}=+1, \\
x_{i} w+b \leq-1, & \text { for } y_{i}=-1 .
\end{array}
$$

That is equivalent to

$$
y_{i}\left[\left(w \bullet x_{i}\right)+b\right]-1 \geq 0, \quad i=1, \cdots, n .
$$

At this point, the classification interval is equal to $2 /\|w\|$, 


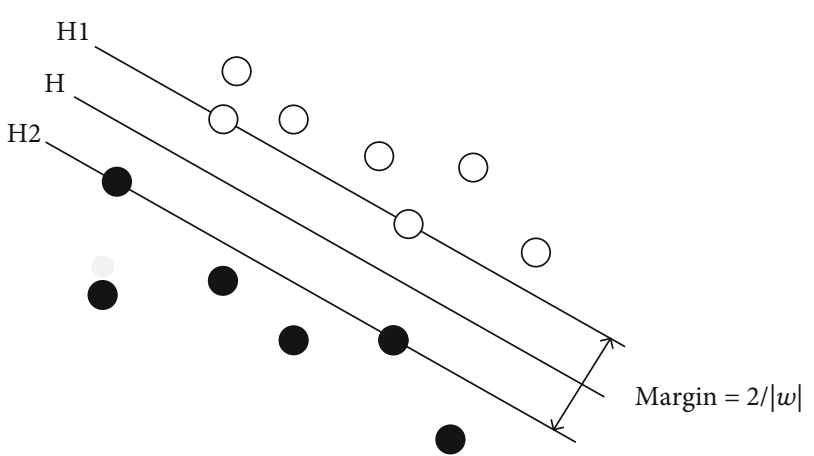

FIGURE 5: Optimal classifier surface in linear condition.

satisfies formula ((2)), and makes it $(1 / 2)\|w\|^{2}$. The smallest classified hyperplane is the optimal hyperplane.H1,H2is called the support vector.

In the case of linear inseparability, a relaxation term can be added to formula (2). $\xi \geq 0$, so the upper form becomes

$$
y_{i}\left[\left(w \bullet x_{i}\right)+b\right]-1+\xi_{i} \geq 0, \quad i=1, \cdots, n .
$$

At this point, we find the minimum $(w, \xi)=(1 / 2)\|w\|^{2}$ $+C\left(\sum_{i=1}^{n} \xi_{i}\right)$ and the generalized optimal classification surface can be obtained. Among them, it is constant and greater than 0 , which controls the degree of punishment of the right and wrong samples.

\subsubsection{Linear Vector Machine. (1) Linear Branchable Support Vector Machine}

Beginning with linear classification, $\left\{x_{i}, y_{i}\right\}, i=1, \cdots, l$, $y_{i} \in\{-1,1\}, x_{i} \in \mathrm{R}^{d}, \quad\left\{x_{i}, y_{i}\right\}, i=1, \cdots, l, y_{i} \in\{-1,1\}, x_{i} \in \mathrm{R}^{d}$, and $\left\{x_{i}, y_{i}\right\}, i=1, \cdots, l, y_{i} \in\{-1,1\}, x_{i} \in \mathrm{R}^{d}$, the above optimal classification surface problem is transformed into a quadratic programming problem:

$$
\begin{array}{ll}
\min & \frac{1}{2}\|w\|^{2} \\
\text { s.t. } & y_{i}\left(w x_{i}+b\right) \geq 1, \quad i=1,2, \cdots, l .
\end{array}
$$

To make the problem easier to deal with, the above problem is transformed into the Lagrange form and the positive Lagrange multiplier $\alpha_{i}, i=1, \cdots, l$ is introduced. Each of them should have an inequality constraint formula (4) for the $\alpha_{i}$ multiplied by positive Lagrange multipliers on both sides of the equation, and approximated by the objective function, the following Lagrange functions are formed:

$$
L_{p}=\frac{1}{2}\|w\|^{2}-\sum_{i=1}^{l} \alpha_{i} y_{i}\left(x_{i} w+b\right)+\sum_{i=1}^{l} \alpha_{i} .
$$

$L_{p}$ must be minimized relative to $\omega, b$, and $L_{p}$ is required to be zero for all derivatives of $\alpha_{i}$ and is bound by $\alpha_{i} \geq 0$ (this special set of constraints is called $C_{1}$ ). To solve the problem, we can solve the dual problem of this problem: $L_{D}$ maximization, subject to $L_{p}{ }^{\prime} s$ constraint on $\omega, b$, and $\alpha_{i} \geq 0$ (this special constraint set is called $C_{2}$ ), and can get the following conditions:

$$
\begin{gathered}
\omega=\sum_{i} \alpha_{i} y_{i} x_{i}, \\
\sum_{i} \alpha_{i} y_{i} x_{i}=0 .
\end{gathered}
$$

Putting the above two generations (6) and (7) into (5) will get

$$
\begin{gathered}
L_{D}=\sum_{i} \alpha_{i}-\frac{1}{2} \sum_{i, j} \alpha_{i} \alpha_{j} y_{i} y_{j}\left(x_{i} \bullet x_{j}\right), \\
\text { s.t. } \quad \alpha_{i} \geq 0, \quad \sum_{i=1}^{l} \alpha_{i} y_{i}=0 .
\end{gathered}
$$

To describe the convenience of the problem, we will refer to $L_{p}$ as the original problem and $L_{D}$ as its dual problem, $L_{p}$ and $L_{D}$ have different constraints, so that $b=0$, then, the hyperplane will pass the origin, and then, $\sum_{i=1}^{l} \alpha_{i} y_{i}=0$ can be eliminated.

According to theKarush-Kuhn-Tuchercondition, the product of the most advantageous Lagrangian multiplier and the constraint are equal to 0 , so the following equation can be obtained.

$$
\alpha_{i}\left[y_{i}\left(w \cdot x_{i}+b\right)-1\right]=0 \text {. }
$$

Regarding any of the standard support vectors, $x_{i}$ will satisfy

$$
y_{i}\left(w \cdot x_{i}+b\right)=1 \text {. }
$$

Then, the parameter $b$ can be calculated by equation (11) as follows:

$$
b=\frac{1}{N_{\mathrm{NSV}}} \sum_{x_{i} \in \mathrm{NSV}}\left(y_{i}-\sum_{x_{j} \in \mathrm{SV}} \alpha_{j} y_{j} x_{j} \cdot x_{i}\right) .
$$

$N_{\text {NSV }}$ is the number of standard support vectors.

After the above discussion, we finally get the discriminant function:

$$
y(x)=\operatorname{sgn}(w \cdot x+b) .
$$

(2) Linear Nonseparable Support Vector Machine

In machine learning, classification and regression analyses are used to analyze data-supervised learning models and related learning algorithms. The above analysis is used to classify the separable data and cannot be applied to the indivisible data. Therefore, we can introduce a loss of $\xi_{i} \geq 0, i=$ $1,2, \cdots, l$, as shown in equation (3). 


$$
\begin{gathered}
\min _{w, b, \xi} \frac{1}{2}\|w\|^{2}+C \sum_{i=1}^{l} \xi_{i} \\
\text { s.t. } \quad y_{i}\left(w \cdot x_{i}+b\right) \geq 1-\xi_{i} .
\end{gathered}
$$

The corresponding $L_{D}$ equation is

$$
\begin{gathered}
L_{D}=\sum_{i} \alpha_{i}-\frac{1}{2} \sum_{i, j} \alpha_{i} \alpha_{j} y_{i} y_{j}\left(x_{i} \cdot x_{j}\right), \\
\text { s.t. } \quad 0 \leq \alpha_{i} \leq C, \quad \sum_{i=1}^{l} \alpha_{i} y_{i}=0 .
\end{gathered}
$$

About $\alpha_{i}$ maximizing $L_{D}$, you can find all $\alpha_{i}, i=1, \cdots, l$.

According to the conditions of formula (6), $\omega=\sum_{x_{i} \in \mathrm{SV}}$ $\alpha_{i} y_{i} x_{i}$ can be obtained. Using equation (11) and according to the Karush-Kuhn-Tucher condition, $b$ can be found, so the classification discriminant $y(x)=\operatorname{sgn}(w \cdot x+b)$.

2.4.3. Nonlinear Support Vector Machine. The classification effect obtained by the linear inseparable support vector machine is not ideal in many cases. Some improvements can be made to the above method, and it is extended to the case where the decision function is not a linear function. By using a nonlinear function $\phi(\cdot)$ to map the sample data $x$ to a high-dimensional linear feature space, $x \longrightarrow \phi(x)$, the discriminant function of the classifier is obtained in a linear space where the dimension may be large and the classification hyperplane is

$$
w \cdot \phi(x)+b=0 \text {. }
$$

And the optimal classification hyperplane is

$$
\begin{gathered}
\min _{w, b, \xi} \frac{1}{2}\|w\|^{2}+C \sum_{i=1}^{l} \xi_{i} \\
\text { s.t. } \quad y_{i}\left(w \cdot \phi\left(x_{i}\right)+b\right) \geq 1-\xi_{i} .
\end{gathered}
$$

In formula (16), $\xi_{i} \geq 0$ is the nonnegative relaxation variable that we introduced, $i=1, \cdots, l$. Dual optimization problem

$$
\begin{gathered}
L_{D}=\sum_{i=1}^{l} \alpha_{i}-\frac{1}{2} \sum_{i=1}^{l} \sum_{j=1}^{l} \alpha_{i} \alpha_{j} y_{i} y_{j} \phi\left(x_{i}\right) \cdot \phi\left(x_{j}\right), \\
\text { s.t. } \quad 0 \leq \alpha_{i} \leq C, \quad \sum_{i=1}^{l} \alpha_{i} y_{i}=0 .
\end{gathered}
$$

For $\alpha_{i}$ to maximize $L_{D}$, all $\alpha_{i}, i=1, \cdots, l$ can be found, thereby finding $\omega=\sum_{x_{i} \in \mathrm{SV}} a_{i} x_{i} y_{i}$ further and finding parameter $b$ according to the Karush-Kuhn-Tucher condition:

$$
b=\frac{1}{N_{\mathrm{NSV}}} \sum_{x_{i} \in \mathrm{NSV}}\left[y_{i}-\sum_{x_{j} \in \mathrm{SV}} \alpha_{j} y_{j} \phi\left(x_{i}\right) \cdot \phi\left(x_{j}\right)\right] .
$$

The above transformation may be complicated and not easy to implement. From equations (14) to (18), it can be found that only the inner product operation $\left(\phi\left(x_{i}\right) \cdot \phi\left(x_{j}\right)\right)$ is involved. According to the related theory of functionals, the kernel function $K\left(x_{i}, x_{j}\right)=\phi\left(x_{i}\right) \cdot \phi\left(x_{j}\right)$ satisfies the Mercer established conditions. That corresponds to the inner product in a transform space. Therefore, $K\left(x_{i}, x_{j}\right)$ can be used instead of inner product to avoid complicated problem solving. The new function is as follows:

From formula (14) to (19), it can be found that all of the above transformations involve only inner product operation $\left(\phi\left(x_{i}\right) \cdot \phi\left(x_{j}\right)\right)$. According to the theory of functional $K\left(x_{i}\right.$, $\left.x_{j}\right)=\phi\left(x_{i}\right) \cdot \phi\left(x_{j}\right)$, as long as a kernel function satisfies Mercer conditions, it corresponds to the inner product in a transformation space. Therefore, $K\left(x_{i}, x_{j}\right)$ can be used instead of the inner product operation to avoid complex problem solving. The new function is as follows:

$$
\begin{gathered}
L_{D}=\sum_{i=1}^{l} \alpha_{i}-\frac{1}{2} \sum_{i=1}^{l} \sum_{j=1}^{l} \alpha_{i} \alpha_{j} y_{i} y_{j} K\left(x_{i}, x_{j}\right), \\
\text { s.t. } \quad 0 \leq \alpha_{i} \leq C, \quad \sum_{i=1}^{l} \alpha_{i} y_{i}=0, \\
b=\frac{1}{N_{\mathrm{NSV}}} \sum_{x_{i} \in \mathrm{NSV}}\left[y_{i}-\sum_{x_{j} \in \mathrm{SV}} \alpha_{j} y_{j} K\left(x_{i}, x_{j}\right)\right] .
\end{gathered}
$$

In summary, the final classification discriminant is

$$
y(x)=\operatorname{sgn}\left(\sum_{i} \alpha_{i} y_{i} K\left(x_{i}, x\right)+b\right) .
$$

The financial risk in $F$ refers to the mark $\Phi(x)$. Assuming that the input data meets the conditions of centralization to guarantee zero mean,

$$
\sum_{k=1}^{M} \phi\left(x_{k}\right)=0
$$

Then, the covariance matrix of the sample in the feature space $F$ is

$$
C=\frac{1}{M} \sum_{k=1}^{M} \phi\left(x_{k}\right) \phi\left(X_{j}\right)^{T}
$$

KPCA does not need to understand the form of the nonlinear mapping function $\Phi$ for feature extraction; only the kernel function needs to be determined.

$$
v \times \phi(x)=\sum_{k=1}^{M} a_{i} \phi\left(x_{k}\right) \phi\left(X_{j}\right)=\sum_{k=1}^{M} a_{i} K\left(x_{i} x\right) .
$$

\subsection{Fuzzy System}

2.5.1. Introduction of Fuzziness. The so-called fuzziness refers to the uncertainty of the nature and scope of objective 


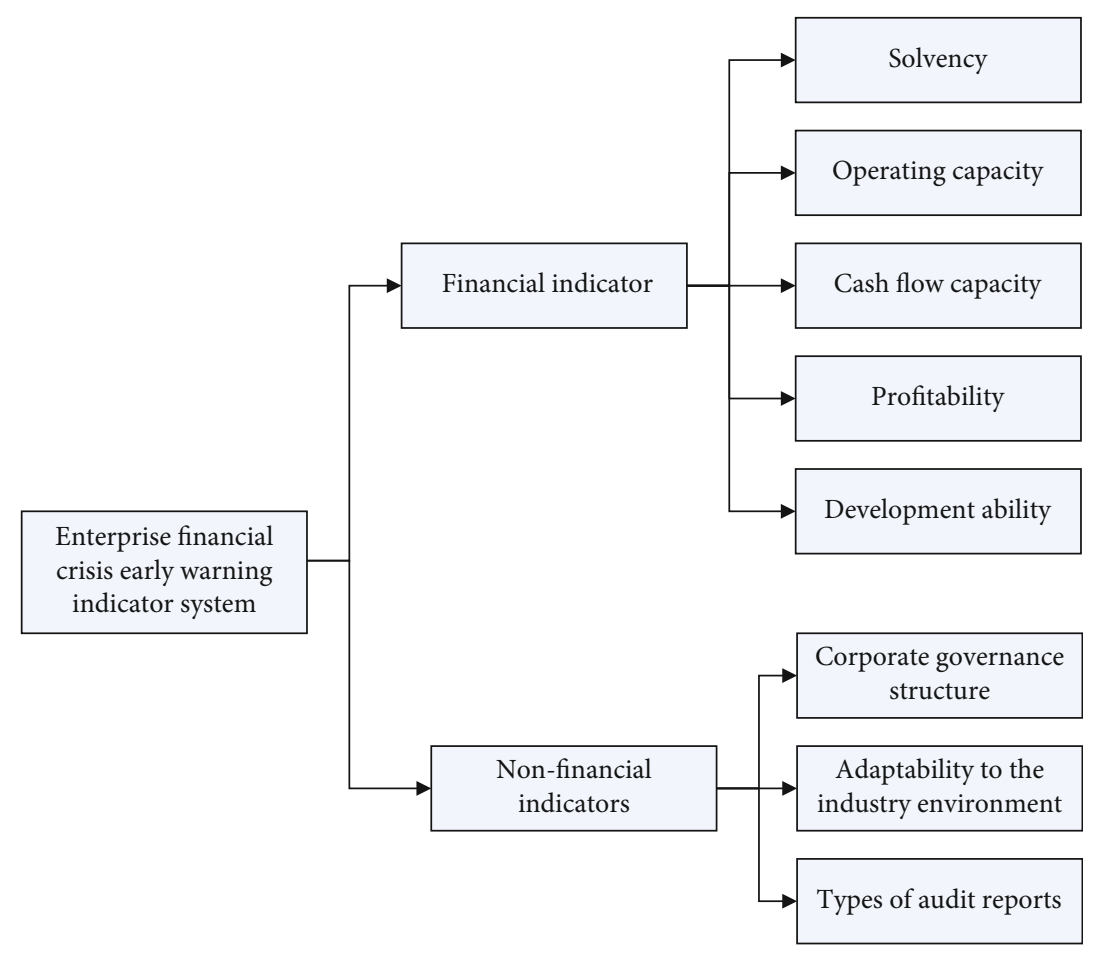

FIGURE 6: Financial crisis SVM early warning indicator system construction framework.

things. Its essence is that there is a series of mutual penetration and interconnection between similar things and there is no clear distinction between them. However, fuzziness is not equal to randomness. It is an uncertain attribute inherent in some objective things, that is, the event itself has uncertainty before it appears. This is caused by the vague concept boundary of the event and the inaccurate definition of the event. Therefore, it has obvious subjective consciousness. Random refers to the uncertainties contained in clearly defined but not necessarily occurring events. It does not change with people's subjective consciousness but is determined by the causal law of things themselves. Fuzzy mathematics does not completely subvert the thought of classical mathematics but takes classical mathematics as the theoretical basis to quantitatively describe and reveal the essence and law of the indefinite and vague things in the objective world.

2.5.2. Fuzzy Statistical Method. Fuzzy statistical decisionmaking refers to a method of finding the optimal strategy in a statistical system with fuzzy information or fuzzy actions. It is actually an extension of classical statistical decision-making. Suppose that $U$ is the domain and $x_{0}$ is a point in $U$. Under the condition that the fuzzy concept is connected, the result of each experiment is to obtain a normal subset $A^{\prime}$ of $U$ and $A^{\prime}$ is related to the fuzzy subset $\tilde{A}$ related. Since each experiment does not strictly define how the fuzzy concept is divided, a different $A^{\prime}$ can be obtained, which may or may not contain $x_{0}$. Due to the uncertainty of the fuzzy concept division, it is impossible to determine the membership relationship of $x_{0}$ to $A^{\prime}$. Therefore, it is necessary to use fuzzy statistics to determine the uncertain prob- lem by means of certain means. It is assumed that in each test, $x_{0}$ is determined and $A^{\prime}$ is constantly changing, $n$ trials are repeated, the membership frequency of $u^{\prime}$ versus $\tilde{A}$ is calculated, and the membership of $u^{\prime}$ to the fuzzy subset $\tilde{A}$ is obtained.

2.5.3. Delphi Method. Using the method of anonymously expressing opinions, through multiple rounds of survey experts' opinions on the questionnaires raised in the questionnaire, after repeated consultations, summarization, and revisions, they are finally summarized into the consensus opinions of the experts. As the result of the prediction, this method has a wide range of representatives. It is more reliable. The Delphi method was originally produced in the field of science and technology and then gradually applied to forecasts in various fields, such as military forecasts, population forecasts, health care forecasts, business and demand forecasts, and education forecasts. In addition, it is also used for evaluation, decision-making, management communication, and planning work. The Delphi method has been widely used in various fields of economic and management science. This method is a typical method to determine the membership degree. Its main idea is to get membership function based on expert's practical experience after certain mathematical treatment. This method can also be used to calculate the fuzzy distribution. In practice, to avoid the different academic level and experience of experts and to have a certain impact on the results, the weighted average method can also be used.

2.6. Financial Early Warning Model. A financial early warning model is a macrofinancial monitoring system that uses a certain statistical method to predict the probability of 


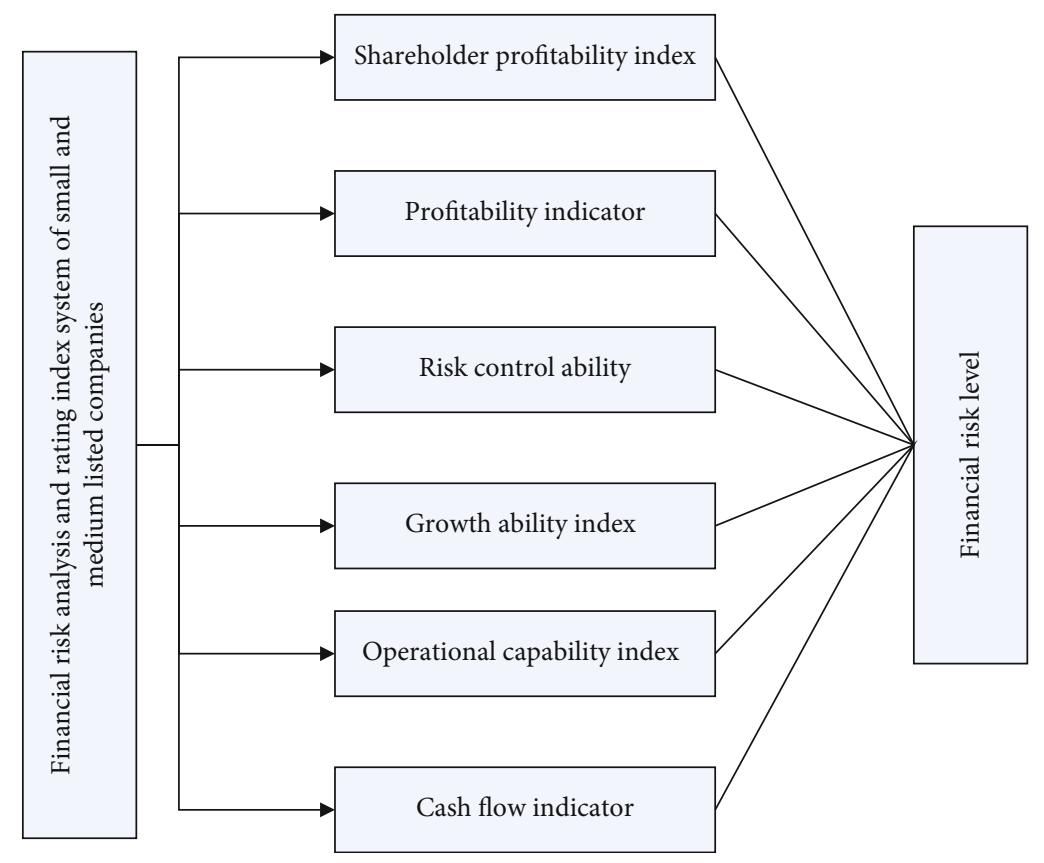

FIGURE 7: Financial risk analysis and rating indicator system diagram of listed companies on the SME board.

currency crises, banking crises, and stock market crashes in a certain economy within a certain time frame. In addition to the indicator system, it also includes major legal frameworks and organizational structures and other constraints. Whether using statistical analysis methods or artificial intelligence methods to establish early warning models, the quality of the selection of financial early warning indicators is directly related to the establishment of early warning models and the accuracy of forecast results. The dotted box in the figure emphasizes the newly added early warning indicators for industry environment adaptation. The construction of the entire early warning indicator system is shown in Figure 6. The symbiosis of the occurrence of multiple types of crises means that there are multiple forms of correlation channels between the various subsystem crises in the financial system, forming a variety of correlation methods and contagion effects. The currency crisis, the banking crisis, the bursting of the bubble in the stock market, and the real estate market are closely linked and contagious and affect the whole body. Therefore, the author believes that when establishing a country's financial risk early warning system, it is necessary to examine the instability factors of the financial system from many aspects according to the market.

Small- and medium-sized enterprises have their own characteristics because they are different from the general main board enterprises, so they comprehensively consider profitability, operating ability, risk control ability, and cash flow ability and increase the two indicators of corporate growth ability and shareholder profitability. Based on the analysis of the overall status quo of listed companies on the SME board, taking into account the feasibility of index data acquisition and quantification, this article is divided into two major levels and six small levels to construct an evaluation index system. The concrete construction idea is shown in Figure 7.
The hardware equipment is shown as in Figure 8.

\section{Experiments}

\subsection{Data Sources}

3.1.1. Selection of Data Sources and Characteristic Indicators. In this paper, a number of small- and medium-sized enterprise companies in a certain economic zone are studied and these companies are treated as special signs of financial crisis due to abnormal financial conditions (ST). Calling it the financial crisis sample company and the financial normal sample company. To obtain as many financial crisis sample companies as possible, this article will be used by ST companies as a financial crisis sample company, with the financial indicators of the previous year of the most recent ST incident as a characteristic indicator, while the remaining other listed companies as a financial indicator. The normal sample company, with its 2018 financial indicators as the characteristic indicators, was finally divided into 28 financial crisis sample companies and 53 financial normal sample companies. In addition, to select as much as possible the characteristics of the listed company's financial status, select a total of 20 financial indicators that reflect the listed company's profitability, development capabilities, solvency, and operational capabilities, as shown in Table 1. The financial data of the sample company is derived from Huatai Joint Stock Trading Software and GOU TAI AN Information Technology Co. Ltd.

3.1.2. Data Preprocessing. Because the financial crisis early warning sample has many characteristic indicators and the dimensions between various indicators are not the same, to avoid the influence of larger dimension indicators on smaller dimensional indicators, this paper uses the standard 


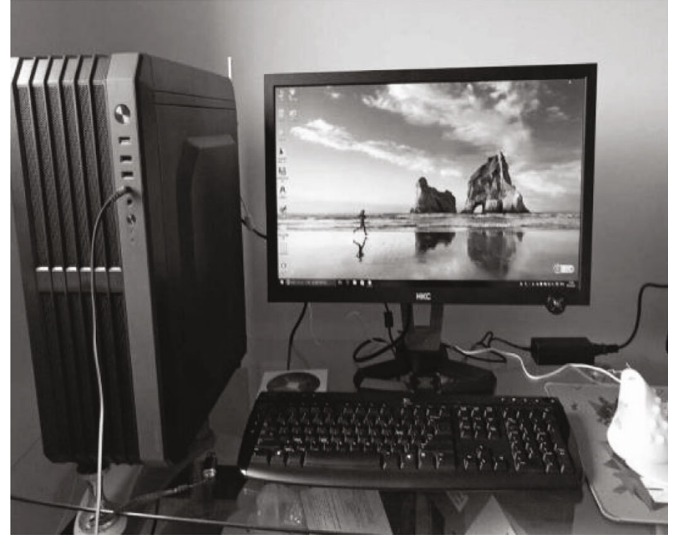

Financial warning operation platform

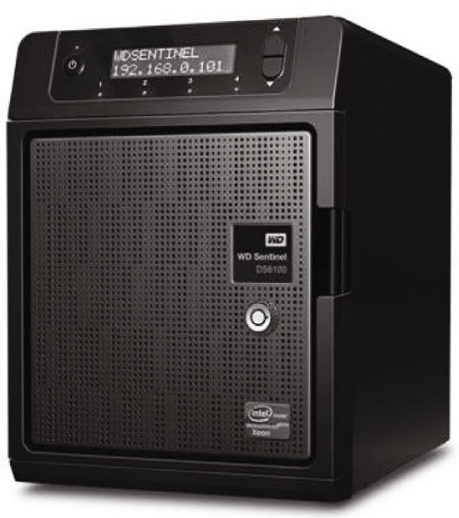

Operating platform server

Figure 8: Financial early warning hardware equipment.

difference method to carry out the innumerable sample data. For outline processing, the method is as follows:

$$
x_{i}^{l}=\frac{\left(X_{i}-\overline{X_{i}}\right)}{\mathrm{SD}_{i}}
$$

Among them, $\overline{x_{i}}$ is the mean of the indicator $x_{i}$ and $\mathrm{SD} i$ is the standard deviation of the indicator $x_{i}$. By performing the above dimensionless processing on the sample data, a new sample set $\left(y_{i}, x_{i}^{1}, s_{i}\right)$ is obtained to further carry out the construction of the FSVM.

\subsection{Training Sample and Test Sample Division. Since the} construction of the financial crisis early warning model needs to be completed through two steps of training and testing, it is necessary to divide the training samples and test samples in advance before constructing the FSVM early warning model. In this paper, $60 \%$ of all financial crisis sample companies and financial normal sample companies are divided into training samples (including 49 listed companies, 35 financial normal sample companies, and 14 financial crisis sample companies), and the remaining $40 \%$ are divided into test samples (including a total of 32 listed companies, 18 financial normal sample companies, and 14 financial crisis sample companies).

3.3. LIBSVM Toolbox. Currently, most people apply the LIBSVM toolbox to classification and regression problems. And LIBSVM has several advantages:

(1) The program is small and requires less computer space

(2) Using the operating default, new default parameters are too many and you need to set the parameters less

(3) Provide the source code, which can be easily extended by those who need it, such as modifying and improving its algorithm in different fields
(4) The calculation takes less time, saves time, and is efficient. Due to its many advantages, it is one of the most widely used SVM libraries in China

More importantly, because it implements various types of files, we can operate the library under Windows. This makes our domestic computers more convenient to use. In summary, the LIBSVM toolbox is used to complete the learning classification and establish a prediction model.

The eigenvalues and contribution rates of each principal component are shown in Table 2.

Selecting the principal components whose eigenvalues exceed 0.65 , there are 7 principal component factors, which contain $89.484 \%$ of the original information. To explain these seven factors, it is necessary to obtain the factor loading of the 14 original financial ratio indicators to these 7 principal component factors (i.e., the correlation coefficient between the original index and the principal component factors). The factor loading matrix is shown in Table 3.

The size of the financing risk is closely related to the business status of the enterprise. From the cash flow statement, borrowing is mainly based on financing but also includes part of external investment. The net cash flow shows that the investment amount is generally high and the inflow of operating activities is generally low. It can be seen that the investment scale of enterprises is gradually expanding, especially in 2017, when the investment amount reached 2.162 billion yuan, while operating activities only generated 1.68100 million yuan. At the same time, the annual amount of debt repayment by enterprises is also huge but it is basically within the controllable range. As shown in Table 4.

The indicators of debt solvency reflect certain financing risks. Only when the company has sufficient working capital to repay its debts can it ensure its healthy development. Three indicators are selected here: asset-liability ratio, current ratio, and quick ratio, as shown in Table 5.

The debt-to-asset ratio is used to measure long-term debt solvency. The debt-to-asset ratio of enterprises has not been high, especially in the past three years, with only about $20 \%$. Regarding the economic situation in recent 
TABLe 1: Financial data of sample companies.

\begin{tabular}{|c|c|c|c|c|c|}
\hline $\begin{array}{l}\text { Financial } \\
\text { capacity }\end{array}$ & $\begin{array}{l}\text { Order } \\
\text { number }\end{array}$ & Financial index & $\begin{array}{l}\text { Financial } \\
\text { capacity }\end{array}$ & $\begin{array}{l}\text { Order } \\
\text { number }\end{array}$ & Financial index \\
\hline \multirow{6}{*}{$\begin{array}{l}\text { Debt paying } \\
\text { ability }\end{array}$} & $\mathrm{X} 1$ & Current ratio & \multirow{6}{*}{ Profitability } & $\mathrm{X} 12$ & Gross profit rate of main business \\
\hline & $\mathrm{X} 2$ & Quick ratio & & $\mathrm{X} 13$ & Profit margin of main business \\
\hline & $\mathrm{X} 3$ & Cash ratio & & $\mathrm{X} 14$ & Cost and expense profit margin \\
\hline & $\mathrm{X} 4$ & Asset-liability ratio & & $\mathrm{X} 15$ & Return on net assets \\
\hline & $\mathrm{X} 5$ & Debt equity ratio & & $\mathrm{X} 16$ & Earnings per share \\
\hline & $\mathrm{X} 6$ & Shareholder equity ratio & & $\mathrm{X} 17$ & All capital earning rate \\
\hline \multirow{4}{*}{$\begin{array}{l}\text { Operational } \\
\text { capacity }\end{array}$} & $\mathrm{X} 7$ & $\begin{array}{c}\text { Average accounts of receivable } \\
\text { turnover ratio }\end{array}$ & \multirow{4}{*}{$\begin{array}{l}\text { Development } \\
\text { ability }\end{array}$} & \multirow[t]{2}{*}{$\mathrm{X} 18$} & \multirow[t]{2}{*}{ Net asset growth rate } \\
\hline & $\mathrm{X} 8$ & Inventory turnover ratio & & & \\
\hline & X9 & Turnover of current assets & & X19 & Total asset growth rate \\
\hline & $\mathrm{X} 11$ & Turnover of total capital & & $\mathrm{X} 20$ & $\begin{array}{c}\text { Growth rate of revenue from main } \\
\text { business }\end{array}$ \\
\hline
\end{tabular}

TABle 2: Table of eigenvalues and contribution rates of each principal component.

\begin{tabular}{lccc}
\hline $\begin{array}{l}\text { Main } \\
\text { ingredient }\end{array}$ & Eigenvalues & $\begin{array}{c}\text { Contribution } \\
\text { rate }\end{array}$ & $\begin{array}{c}\text { Total contribution } \\
\text { rate }\end{array}$ \\
\hline 1 & 4.428 & 31.630 & 31.630 \\
2 & 2.707 & 19.339 & 50.969 \\
3 & 2.046 & 14.615 & 65.584 \\
4 & 964 & 6.883 & 72.467 \\
\hline
\end{tabular}

years, maintaining a low debt ratio is indeed conducive to coping with risks, but enterprises must also actively use leverage to obtain more benefits for enterprises, as shown in Figure 9.

Investment activities will bring investment risks. Fixed assets and intangible assets account for a large proportion of Neusoft's inward investment. Foreign investment involves three subjects: investment real estate, long-term equity investment, and available-for-sale financial assets, as shown in Table 6.

The amount of inward investment by enterprises does not increase steadily but has experienced a trend of rising first, then falling and then rising. Among them, due to the continuous completion of the project, the construction in progress has been continuously transferred to fixed assets. Also because of the continuous advancement of the R\&D process, $\mathrm{R} \& \mathrm{D}$ expenditures are gradually transferred to intangible assets. However, because the large randomness of whether $\mathrm{R} \& \mathrm{D}$ projects in the information technology industry can be successfully realized, there is a large instability in the inward investment activities of enterprises, as shown in Table 7.

The growth of corporate outbound investment is particularly prominent in available-for-sale financial assets. The shares subscribed for in 2017 include Honghe Renai Medical Co. Ltd. and the Bank of Gansu, which were all sold at the initial public offering price. In 2018, it subscribed for the initial public offering of AsiaInfo Technologies with its own funds. Due to the existence of other price risks, there are many unstable factors for Neusoft Group to subscribe for shares and foreign investment risks appear, as shown in Table 8.

If an enterprise has a high level of profitability, it indicates that it is capable of repaying debts with profitable funds, without the need to sell or mortgage its own assets. Here, four indicators of the operating profit rate, cost and expense profit rate, return on total assets, and return on net assets are selected for analysis, as shown in Table 9.

Return on net assets reflects the profitability of owners' equity. The company's return on net assets is above the industry average, but the industry is only at the middle and lower reaches of the industry, and its profit prospects are not optimistic, as shown in Figure 10.

The operational risks of an enterprise have appeared in the indicators of operational capability, and the stable development of an enterprise depends on a strong operational capability. Here, three indicators of the current asset turnover rate, total asset turnover rate, and accounts receivable turnover rate are selected to analyze the operating capability, as shown in Table 10.

The net profit has shown a trend of rapid increase and sharp decline, which shows the determination of the enterprise to reorganize in recent years. In particular, the company's loss of control over Neusoft Medical and Xikang in 2016 caused a substantial increase in profits. However, the distribution of cash dividends is still in a relatively stable state and there has been no significant increase or decrease in recent years, which helps in enhancing shareholders' trust in the company. At the same time, the distribution of Neusoft's cash dividends in 2018 is also worth looking forward to. In the face of negative net profit, it is obvious that the risk of income distribution has increased, as shown in Table 11.

The change in the growth rate of net assets reflects the state of the company's expansion in 2015, 2016, and 2017. In 2018, the capital has shown a negative growth trend. It is very likely that the company does not have enough funds to supply the expansion of the scale and even bring cash flow 
TABLE 3: Factor loading matrix.

\begin{tabular}{lccccc}
\hline 一 & Component 1 & Component 2 & Component 3 & Component 4 & Component 5 \\
\hline X1 & 0.368 & 0.720 & 0.292 & 0.044 & 0.246 \\
X2 & 0.373 & 0.486 & 0.365 & 0.328 & 0.505 \\
X3 & 0.457 & 0.701 & 0.312 & 0.137 & 0.057 \\
X4 & 0.608 & 0.072 & 0.750 & 0.188 & 0.002 \\
\hline
\end{tabular}

TABLE 4: 2015-2018 cash flow partial statement.

\begin{tabular}{|c|c|c|c|c|}
\hline Report date & $2018-12-31$ & $2017-12-31$ & $2016-12-31$ & $2015-12-31$ \\
\hline Net cash flow from operating activities & 3.28 & 1.68 & 1.47 & 2.87 \\
\hline Net cash flows from investing activities & -0.74 & -21.62 & -10.58 & -6.45 \\
\hline Absorb cash received from investment & 2.84 & 15.11 & 11.12 & 8.92 \\
\hline Obtain the cash received from the loan & 9.24 & 7.71 & 19.71 & 27.81 \\
\hline
\end{tabular}

TABLE 5: 2015-2018 changes in solvency.

\begin{tabular}{lcccc}
\hline Report date & $2018-12-31$ & $2017-12-31$ & $2016-12-31$ & $2015-12-31$ \\
\hline Current ratio & $1.6 \%$ & $2.19 \%$ & $2.17 \%$ & $1.61 \%$ \\
Quick ratio & $1.24 \%$ & $1.79 \%$ & $1.82 \%$ & $1.32 \%$ \\
Assets and liabilities & $33.6 \%$ & $29.75 \%$ & $31.45 \%$ & $47.23 \%$ \\
\hline
\end{tabular}

risk. The growth rate of the company's total assets shows a fluctuating development trend, which may be related to the company's annual strategic development, but there is a suspicion of blind expansion. It is necessary to track the quality of asset expansion and whether the follow-up development momentum is sufficient. The growth rate from $25.42 \%$ to $456.26 \%$ and then from $456.26 \%$ to $-47.78 \%$ is a little bit hit in this span; this is very likely to have fraudulent accounts, as shown in Figure 11.

\section{Discussion}

4.1. Comparative Analysis of Different Kernel Functions in Experiments. This article uses MATLAB2011b to model, SVM parameter $C$ is set to 1 , and other parameters take the default settings. The comparison between the FCMSVM and the SVM models under the four kernel functions is shown in Figure 12. It can be clearly seen in Figure 2 that the prediction accuracy of the FCM-SVM model is much higher than that of SVM, no matter which kernel function is used, which indicates that the fuzzy method can effectively reduce the impact of singular points or wild spots in the sample on SVM prediction performance, thereby greatly improving the prediction accuracy of the SVM. In addition, it is found that different kernel functions have different effects on the prediction performance of the FCM-SVM model. Under the Gauss radial basis kernel function, the prediction accuracy of the FCM-SVM is as high as $86 \%$, which is much higher than the FCM of the other three kernel functions. The prediction accuracy of SVM indicates that the FCM-SVM model under the Gauss radial basis kernel function has the most superior predictive performance in the financial crisis prediction of small- and medium-sized listed companies in the economic zone.

4.2. Comparative Analysis of Performance of Different Early Warning Models. In addition, to more fully demonstrate the superior predictive performance of the FCM-SVM model, this paper also uses the BP neural network (BPNN), decision tree (DT), logit regression and probit regression four models, and Gauss radial basis kernel function. The FCM-SVM was compared and the results are shown in Figure 13. The prediction accuracy of the FCM-SVM is as high as $86.69 \%$, BPNN is $52.17 \%$, DT is $26.09 \%$, and LOGIT and probit are both $82.61 \%$. It can be seen from this that the prediction accuracy of the FCM-SVM is good. As can be seen in Figure 3, the FCM-SVM has the highest prediction accuracy, indicating that the FCM-SVM is not only superior to traditional early warning models, such as logit and probit, but also better than the other intelligent early warning models, such as BPNN and DT. The FCM-SVM model with Gauss radial basis kernel function has the most superior prediction performance.

4.3. Analysis of McNemar Test Results for Different Early Warning Models. Since the prediction performance of the model is only lacking from the classification accuracy rate, it lacks the scientificity similar to the mathematical statistics test. Therefore, this paper will introduce the McNemar test method to compare the prediction results of each early warning model to see if there is a significant difference, which is more scientific. The predicted performance of each model was accurately evaluated, and the results are shown in Figure 14. It can be seen in Figure 4 that the McNemar test $p$ values of the FCM-SVM and the other four models are all 


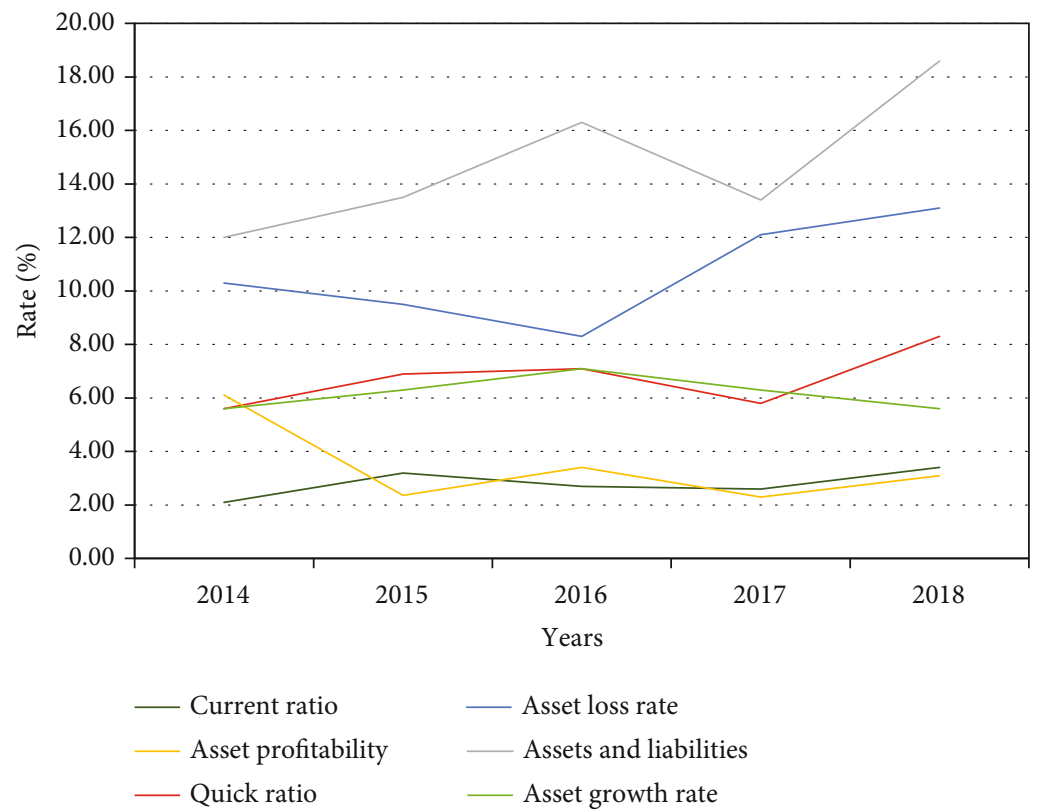

Figure 9: Chart of changes in debt solvency from 2014 to 2018.

TABLE 6: 2015-2018 inward investment situation table.

\begin{tabular}{lcccc}
\hline Report date & $2018-12-31$ & $2017-12-31$ & $2016-12-31$ & $2015-12-31$ \\
\hline Fixed assets & $1,769,954,205$ & $1,607,798,554$ & $1,479,246,234$ & $1,812,379,068$ \\
Construction in progress & $126,291,398$ & $146,943,581$ & $346,179,321$ & $477,054,653$ \\
Intangible assets & $590,371,127$ & $551,113,962$ & $643,101,930$ & $755,271,986$ \\
\hline
\end{tabular}

TABLE 7: Table of inward investment growth rate, etc.

\begin{tabular}{lcccc}
\hline Report date & $2018-12-31$ & $2017-12-31$ & $2016-12-31$ & $2015-12-31$ \\
\hline R\&D spending & $1,769,954,205$ & $1,607,798,554$ & $1,479,246,234$ & $1,812,379,068$ \\
Other expenses & $253,881,787$ & $422,147,331$ & $2532,623,080$ & $94,058,499$ \\
Growth rate & $9.57 \%$ & $-4.36 \%$ & $-18.15 \%$ & $5.36 \%$ \\
\hline
\end{tabular}

TABLE 8: 2015-2018 foreign investment situation table.

\begin{tabular}{|c|c|c|c|c|}
\hline Report date & $2018-12-31$ & $2017-12-31$ & $2016-12-31$ & $2015-12-31$ \\
\hline Available for sale of financial assets & $197,197,037$ & $38,245,830$ & $5,853,340$ & $35,678,420$ \\
\hline Long-term equity investment & $3,079,630,695$ & $2,966,950,050$ & $793,382,049$ & $232,547,310$ \\
\hline Investment real estate & $856,127,717$ & $893,389,082$ & $600,607,506$ & $620,933,753$ \\
\hline
\end{tabular}

TABLE 9: 2015-2018 profitability change table.

\begin{tabular}{lcccc}
\hline Report date & $2018-12-31$ & $2017-12-31$ & $2016-12-31$ & $2015-12-31$ \\
\hline ROA & -0.76 & 6.9 & 14.51 & 2.53 \\
Cost and expense margin & -0.82 & 14.72 & 21.43 & 5.22 \\
Operating profit margin & -0.84 & 13.27 & 24 & 1.77 \\
ROE & 1.25 & 11.87 & 6.36 \\
\hline
\end{tabular}




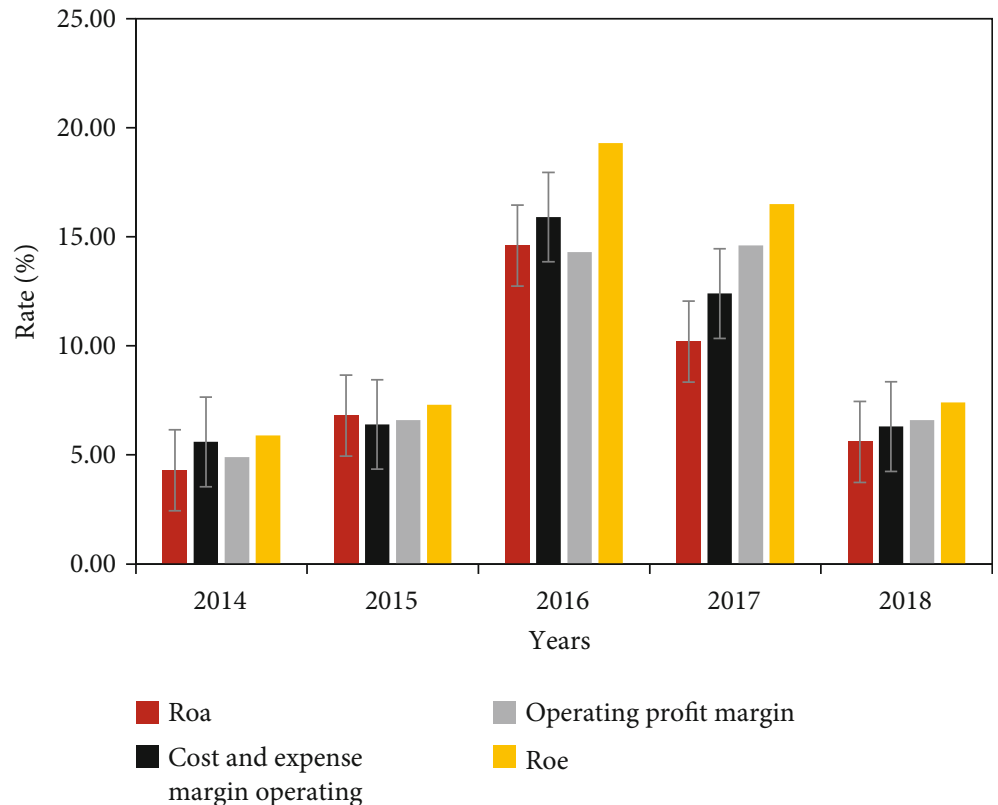

FIgURE 10: Trend of profitability changes from 2014 to 2018.

TABLE 10: 2015-2018 operating capability change table.

\begin{tabular}{|c|c|c|c|c|}
\hline Report date & $2018-12-31$ & $2017-12-31$ & $2016-12-31$ & $2015-12-31$ \\
\hline Accounts receivable turnover rate & 4.12 & 4.66 & 4.29 & 3.74 \\
\hline Turnover rate of total assets & 0.54 & 0.58 & 0.65 & 0.71 \\
\hline Liquid asset turnover rate & 1.14 & 1.14 & 1.11 & 1.2 \\
\hline
\end{tabular}

TABle 11: 2015-2018 income distribution.

\begin{tabular}{lcccc}
\hline Report date & $2018-12-31$ & $2017-12-31$ & $2016-12-31$ & $2015-12-31$ \\
\hline Net profit & $-103,289,428$ & $890,223,697$ & $1,704,646,737$ & $306,447,734$ \\
Growth rate & $-111.60 \%$ & $-47.78 \%$ & $456.26 \%$ & $25.42 \%$ \\
Cash dividend & - & $111,841,020$ & $124,319,775$ & $118,044,791$ \\
Growth rate & - & $-10.04 \%$ & $5.32 \%$ & $52.63 \%$ \\
\hline
\end{tabular}

less than $1 \%$, indicating that the significance of the FCMSVM and the other four models is proved by the significance test with a significance level of $1 \%$. There are significant differences between performance.

In summary, under the four kernel functions, the prediction performance of the FCM-SVM model is better than that of the SVM model, especially that the FCM-SVM model under the Gauss radial basis kernel function has the most superior prediction performance and is also significantly superior. And it is also significantly superior to traditional statistical models and other artificial intelligence early warning models.

4.4. Logistic Model Analysis. Compared with the completion of the efficiency coefficient method, the more important thing is the establishment of evaluation standards, which determine the division of corporate financial risks. Due to the poor flexibility of risk division originally stipulated by the efficacy coefficient method, this article uses five levels to classify financial risks after referring to the previous classifications, namely, nonalert, mild, moderate, severe, and giant, as shown in Table 12.

After the above experimental steps, the statistics of 100 training and test results in each case are as follows and the training and test accuracy is retained two decimal places, as shown in Table 13.

The sample number of companies that have declared bankruptcy or delisting as distressed companies is 32 . Four classification models are used to classify financial data. The classification results are shown in Table 14.

To make the results of this paper more convincing, the collinearity test will be carried out after statistical test according to the index system of "asset scale," and then, the logistic regression model will be established directly after 


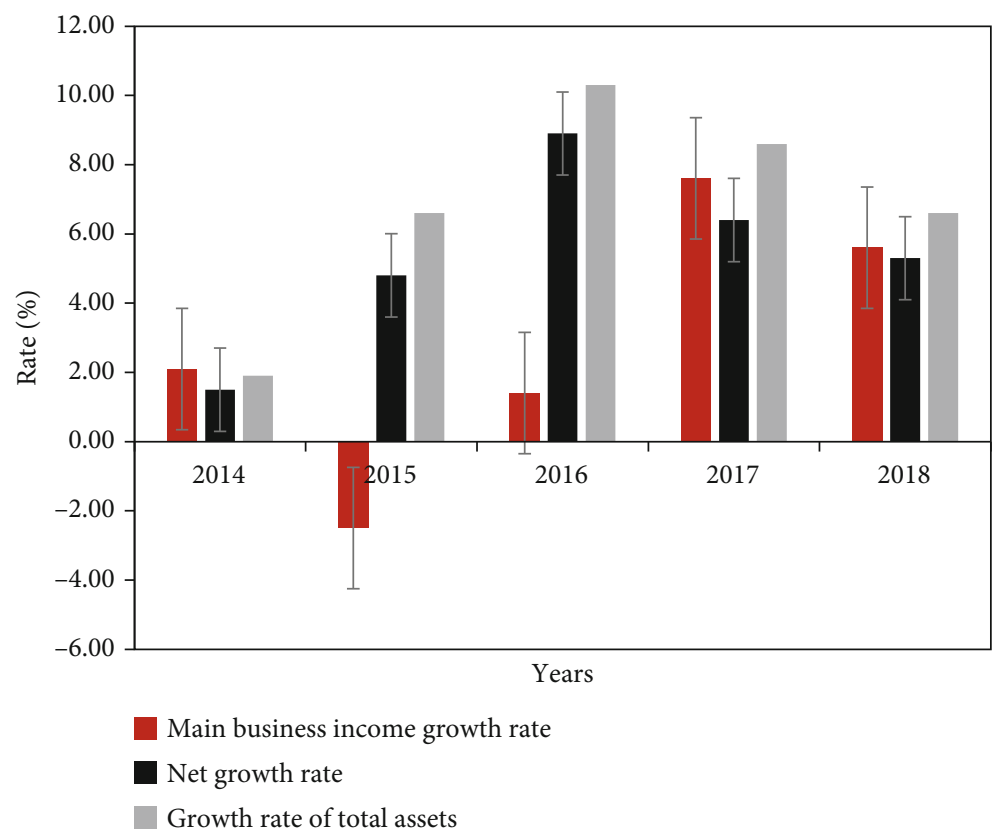

FIGURE 11: Development capacity change trend graph from 2014 to 2018.

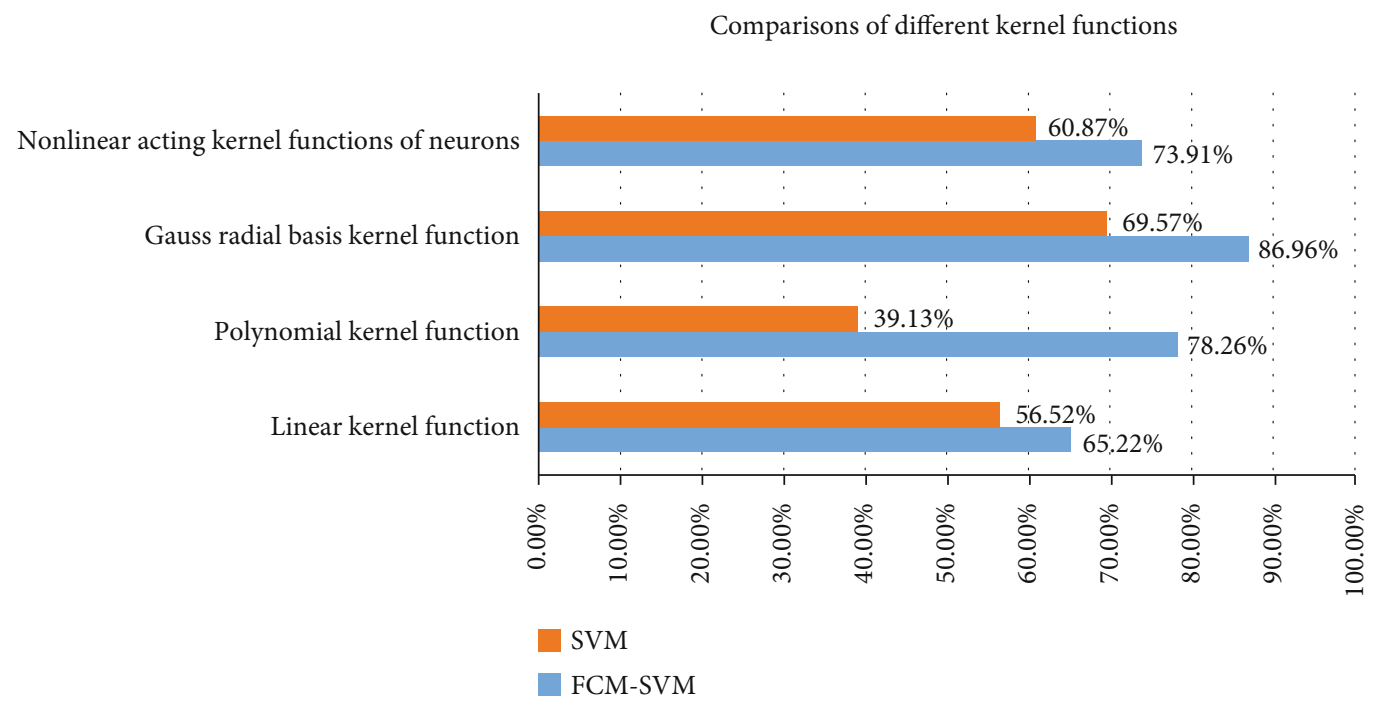

Figure 12: Comparison of different kernel functions.

standardization and normalization and the tenfold crossover will be carried out. The prediction results are shown in Table 15 and Figure 15.

It can be seen from the experimental results in Table 2 and Figure 5 that the logistic model will be directly established by the financial indicator system that passed the significance test and the collinearity test. The prediction accuracy percentages in T-2, T-3, and T-4 are 90\%, $71.25 \%$, and $57.5 \%$, respectively, and on the basis of the addition of corporate governance indicators, the forecast accuracy has improved significantly, followed by $91.25 \%$, $72.5 \%$, and $60 \%$ respectively. Compared with the classical logistic experiment results, the logistic regression model established after RS reduction has significantly improved the accuracy of SME financial crisis early warning classification. The prediction accuracy rates in T-2, T-3, and T-4 are $91.25 \%, 71.25 \%, 65 \%$, respectively; after adding corporate governance indicators, the forecast accuracy rates are $93.75 \%, 73.75 \%$, and $66.25 \%$, respectively. That is to say, the logistic regression model is established directly by using the statistical test index system and its prediction accuracy is lower than that of the model based on the RS reduction index system.

4.5. Discussion on the Causes of Financial Crisis. China's small- and medium-sized listed companies are mostly small and growing small enterprises. Compared with many large enterprises, they have the following characteristics: firstly, 


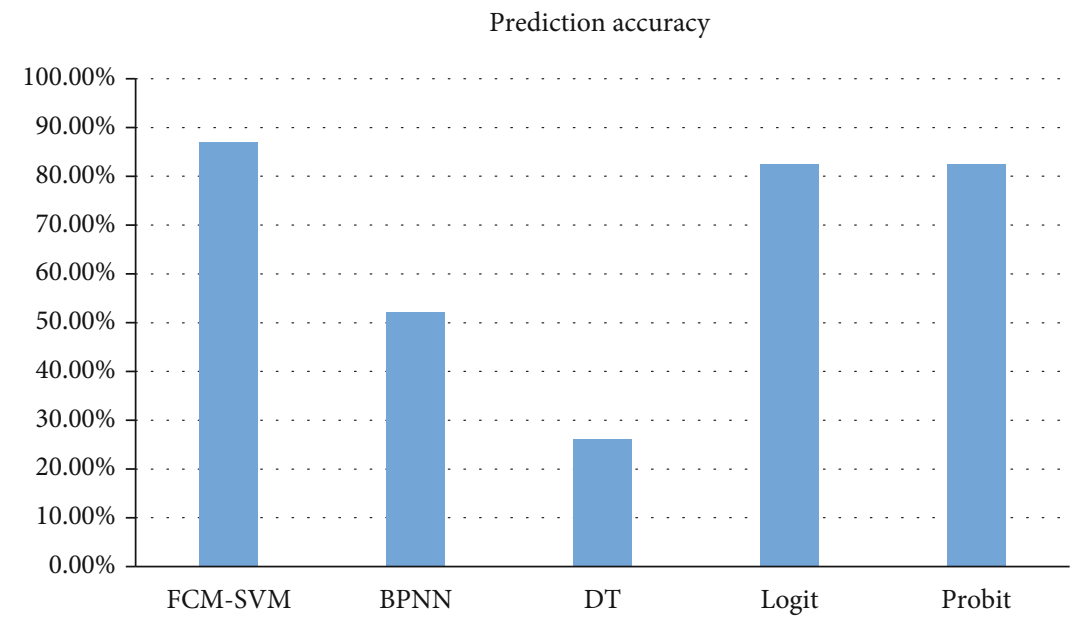

FIGURE 13: Performance comparison results of different early warning models.

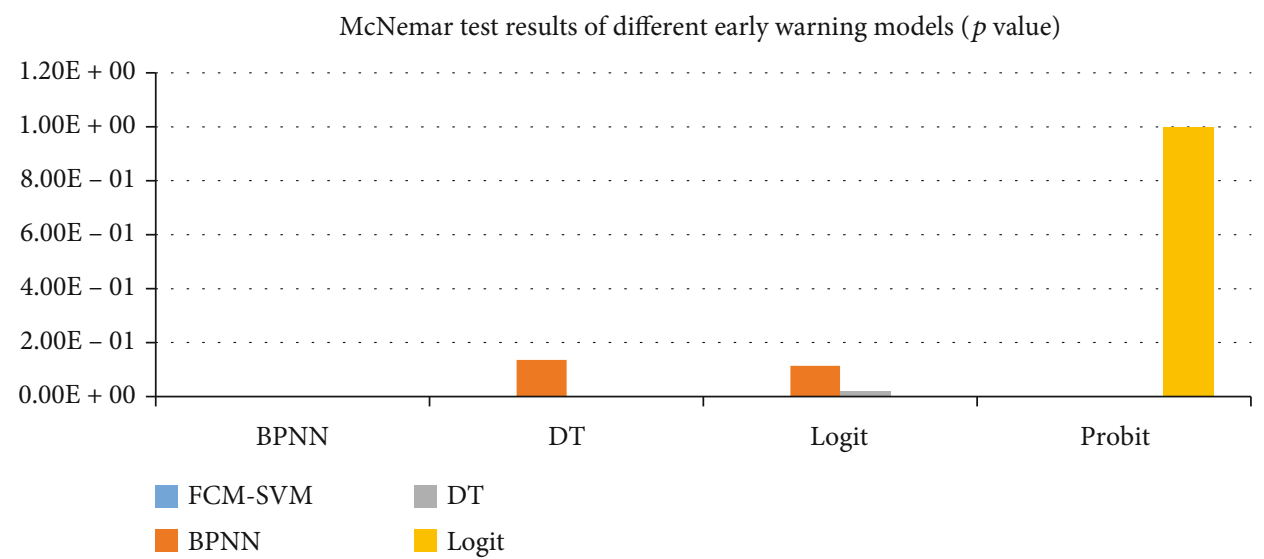

Figure 14: McNemar test results ( $p$ values) for different early warning models.

TABLE 12: Financial risk warning levels.

\begin{tabular}{|c|c|c|c|c|}
\hline Warning level & Evaluation interval & \multicolumn{2}{|c|}{ Risk profile } & \\
\hline Giant police & $0 \leq X<0.3$ & \multicolumn{2}{|c|}{ Significant risk } & $\begin{array}{l}\text { The } \\
\text { on the }\end{array}$ \\
\hline Heavy warning & $0.3 \leq X<0.5$ & \multicolumn{2}{|c|}{ Greater risk } & The o \\
\hline Police & $0.5 \leq X<0.7$ & \multicolumn{2}{|c|}{ Less risk } & The bu \\
\hline Light police & $0.7 \leq X<0.85$ & \multicolumn{2}{|c|}{ Focus on risk } & The o \\
\hline No police & $0.85 \leq X<1$ & \multicolumn{2}{|c|}{ No risk } & $\begin{array}{r}\text { The bus } \\
\text { situati }\end{array}$ \\
\hline \multicolumn{5}{|c|}{ TABLE 13: Financial risk early warning training test situation. } \\
\hline \multicolumn{3}{|l|}{ Training set/test set } & \multicolumn{2}{|c|}{ Total test accuracy } \\
\hline $8 / 2$ & \multicolumn{2}{|l|}{$85.08 \%$} & \multicolumn{2}{|c|}{$73.51 \%$} \\
\hline $7 / 3$ & \multicolumn{2}{|l|}{$84.97 \%$} & \multicolumn{2}{|c|}{$73.21 \%$} \\
\hline $6 / 4$ & \multicolumn{2}{|l|}{$85.16 \%$} & \multicolumn{2}{|c|}{$73.64 \%$} \\
\hline
\end{tabular}

The financial situation of the enterprise has fallen to the bottom and is any time. Almost all indicators show a negative trend

The operation of the enterprise is difficult, most of the financial indicators are at the lowest value, and it is very likely to face financial risks

The business is not doing well, some indicators are deteriorating, the overall financial situation is declining, and it may face financial risks

he operation of the enterprise is almost normal, and individual indicators are abnormal, and there is little possibility of facing financial risks situation is stable, and there is only a small chance of facing financial risks
siness is operating well, almost all indicators perform well, the financia

they are small in scale. The small scale of production and operation is the primary feature of small- and mediumsized listed companies that are different from large enterprises. Due to the limited resources necessary for the operation of small- and medium-sized listed companies, such as manpower, material resources, and financial resources, small- and medium-sized listed companies generally have a relatively simple business model, relatively large operational 
TABLE 14: Classification comparison of the test set under different algorithms.

\begin{tabular}{lccccc}
\hline Algorithm & nSV & Category 1 (\%) & Category 2 (\%) & Category 3 (\%) & Accuracy (\%) \\
\hline SAPSO-FSVM & 88 & $84.6 \%$ & $79.0 \%$ & $62.5 \%$ & $79.29 \%$ \\
PSO-FSVM & 127 & $74.0 \%$ & $75.8 \%$ & $65.6 \%$ & 73.235 \\
GA-FSVM & 107 & $71.2 \%$ & $82.3 \%$ & $59.4 \%$ & $72.73 \%$ \\
FSVM & 195 & $73.1 \%$ & $43.5 \%$ & $68.8 \%$ & $68.18 \%$ \\
\hline
\end{tabular}

TABLe 15: Comparison of experimental results between the logistic model and the RS-logistic model.

\begin{tabular}{lcrr}
\hline & Datasets & Logistic & RS-logistic \\
\hline T-2 & Financial index & $0.900 \pm 0.1291$ & $0.9125 \pm 0.0645$ \\
& Financial corporate governance index & $0.912 \pm 0.1186$ & $0.9375 \pm 0.0645$ \\
\hline T-3 & Financial index & $0.712 \pm 0.1186$ & $0.7125 \pm 0.0800$ \\
& Financial corporate governance index & $0.725 \pm 0.1021$ & $0.7375 \pm 0.1281$ \\
T-4 & Financial index & $0.575 \pm 0.1687$ & $0.6500 \pm 0.0935$ \\
& Financial corporate governance index & $0.600 \pm 0.1748$ & $0.6625 \pm 0.1256$ \\
\hline
\end{tabular}

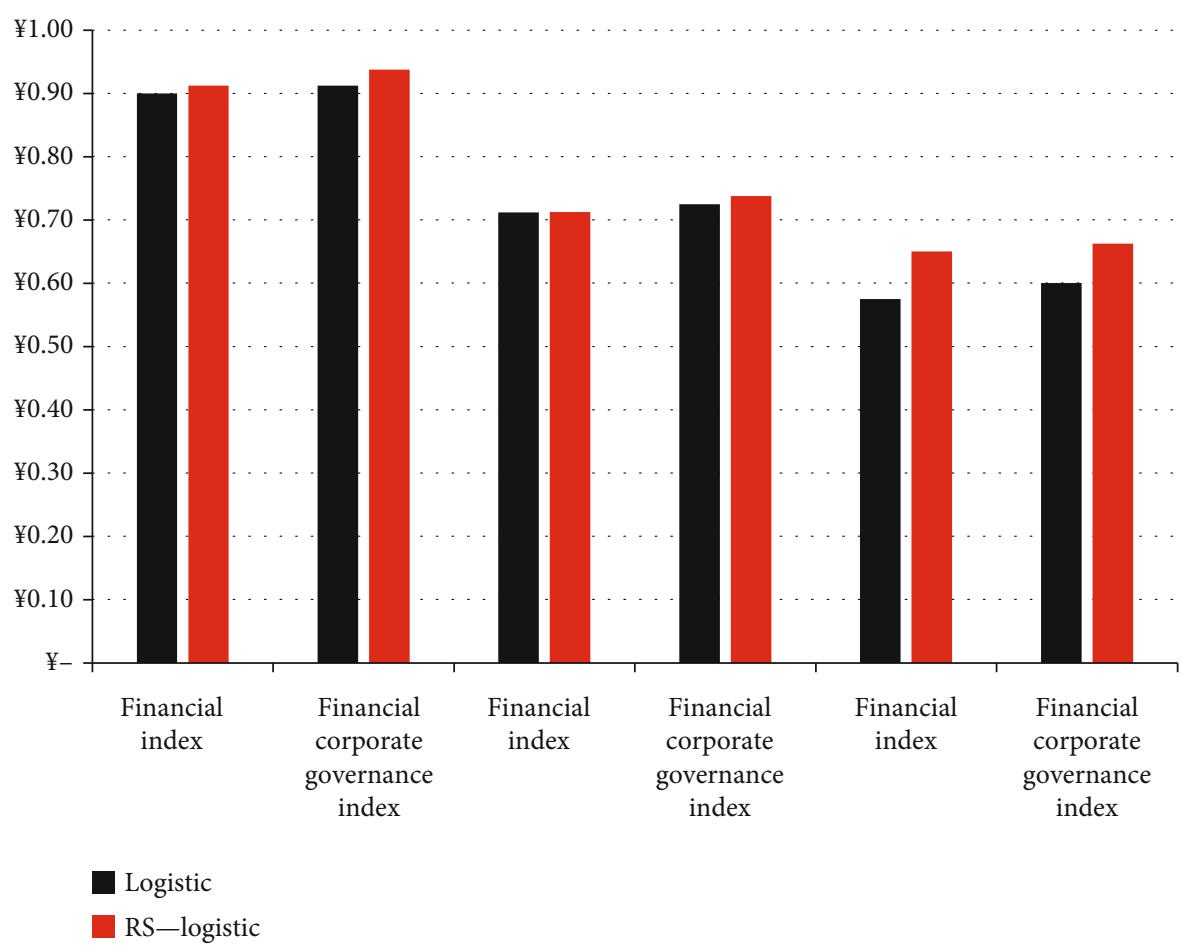

FIGURE 15: Comparison of experimental results between the logistic model and RS-logistic model.

risks, and relatively low market share. Second is the number. Small- and medium-sized listed companies have low barriers to enter in the market, so the number is large. With the support of the national policy environment, the distribution of small- and medium-sized listed companies and the industries involved have gradually expanded and become an important force in the development of the national economy. Furthermore, ownership is highly concentrated. Most small- and medium-sized listed companies are funded by one or several investors, so the decision-making power is more concentrated and the management decision-making process is faster. Finally, the bankruptcy rate is high. The establishment of small- and medium-sized listed companies in China has fewer restrictions. The operators are mostly individual investors, lack the quality of professional managers, and have less long-term planning. Therefore, they are more sensitive to the development of the economic environment and have relatively weaker ability to resist market risks.

Therefore, the causes of the financial crisis of small- and medium-sized listed companies can be discussed and analyzed from several aspects: first, the capital structure is 
unreasonable. Small- and medium-sized listed companies are mostly small and emerging enterprises, lack of capital structure and financial risk measurement, have less longterm planning, and have blind investment. In addition, the relative centralization of decision-making power in operation and management increases the risk of overinvestment or underinvestment, resulting in financial risks due to unreasonable capital structure. Second is financing difficulties. There are a large number of small- and medium-sized listed companies in China, which are established quickly, but there are also many small- and medium-sized listed companies that fail. One of the key reasons is the financing problem. Due to the unreasonable governance structure and blind investment of small- and medium-sized listed companies, the financial risk of enterprises is high and investors lack confidence in the investment of small- and medium-sized enterprises, which leads to the financing difficulties of smalland medium-sized enterprises. Once the enterprise capital chain is interrupted, the enterprise will fall into a greater crisis and it is difficult to reverse. Third, the governance structure is unreasonable. Due to the low entry threshold, many investors and corporate managers do not have the quality of professional managers, corporate management is not standardized, the internal management system of employees is not perfect, and the application of financial systems is unreasonable. This series of problems will lead to poor business operations, weak ability to bear risks, and being prone to financial crises. Fourthly, there is a lack of effective financial crisis early warning mechanism. Compared with postcontrol, precontrol is more effective and cost effective. The operators of small- and medium-sized listed companies lack risk prevention awareness, fail to establish an effective financial crisis early warning system, and fail to find problems in operation in time and take corresponding preventive measures, resulting in frequent financial crises of small- and medium-sized listed companies.

\section{Conclusions}

This paper takes the small- and medium-sized listed companies in the economic zone as the research object, introduces the fuzzy method into the SVM, constructs the FSVM model, and makes a performance comparison study based on four different kernels. At the same time, it also makes a performance comparison study with the traditional statistical model and other artificial intelligence models. The empirical results show that the FSVM model based on Gauss radial basis function has the best prediction performance compared with the other three kernel functions. At the same time, it is also significantly superior to the traditional statistical model and other artificial intelligence models. Based on the above empirical results, this paper argues that the FSVM model under Gauss radial basis function can most effectively predict the financial crisis of small- and medium-sized companies in China's economic zone, which can play a good role in maintaining the stable development of the economic zone.

After analyzing the advantages and disadvantages of different stock investment models, this paper chooses the
SVM integrated model to study stock selection. According to the defects of the previous SVM integrated model in application, this paper explains the necessity of improving the SVM-integrated model and introduces the variable penalty factor and fuzzy membership degree to improve the traditional SVM integrated model. Finally, a fuzzy dynamic SVM integrated model is constructed, which makes it possible to select different penalty factors for different types of stocks instead of simply dividing them into two equal categories. The model can be directly applied to the prediction of annual return on investment of stocks and improve the application of the SVM in the field of stock investment.

This paper successfully constructed an evaluation index system for wireless network communication and the application of the optimal fuzzy SVM artificial intelligence model. Based on the quarterly report data of 2018 of several smalland medium-sized listed companies in an economic zone, four groups of experiments in different industries were designed with the annual return on investment as the predictive variable. Factor analysis is used for dimensionality reduction. By compiling LIBSVM and running on WEKA, the results of stock selection of the integrated model of the fuzzy dynamic SVM are output. The investment returns of the selected results are higher than the average return of the sample. It is proved that the wireless network communication and optimal fuzzy SVM artificial intelligence model established in this paper can be used for investors to make further investment decisions. Under the Gaussian radial basis kernel function, the prediction accuracy of the FCMSVM is as high as $86 \%$, which is much higher than that of the FCM of the other three kernel functions. The prediction accuracy of the SVM shows that the FCM-SVM model under the Gaussian radial basis kernel function has the most superior prediction performance in the financial field. Through comparative analysis of the integration of fuzzy dynamic support vector machines and traditional support vector machines, fuzzy dynamic support under different sample sizes is obtained. Compared with the support vector machine integration model, the prediction error of the vector machine integration model has fewer industries and time conditions, which proves that the improvement of wireless network communication and the optimal fuzzy SVM artificial intelligence model is effective. There are still many unresolved problems in the theory and technology of the article, and the consideration of the problems is not comprehensive. But I believe that in the future research life, we will make financial early warning models more widely used and more valuable.

\section{Data Availability}

This article does not cover data research. No data were used to support this study.

\section{Conflicts of Interest}

The authors declare that they have no conflicts of interest. 


\section{References}

[1] A. H. Ayob, S. Ramlee, and A. Abdul Rahman, "Financial factors and export behavior of small and medium-sized enterprises in an emerging economy," Journal of International Entrepreneurship, vol. 13, no. 1, pp. 49-66, 2015.

[2] R. Forte and A. S. Moreira, "Financial constraints and small and medium-sized firms' export propensity: evidence from Portuguese manufacturing firms," International Journal of the Economics of Business, vol. 25, no. 2, pp. 223-241, 2018.

[3] J. Haselip, D. Desgain, and G. Mackenzie, "Non-financial constraints to scaling-up small and medium-sized energy enterprises: findings from field research in Ghana, Senegal, Tanzania and Zambia," Energy Research \& Social Science, vol. 5, no. 6, pp. 78-89, 2015.

[4] B. Zheng and D. Yun, "A wireless network communication capacity control technology based on fuzzy wavelet neural network," Wireless Communications and Mobile Computing, vol. 2021, no. 7, Article ID 9994200, 10 pages, 2021.

[5] D. Fernández-Arias, M. López-Martín, T. Montero-Romero, F. Martínez-Estudillo, and F. Fernández-Navarro, "Financial soundness prediction using a multi-classification model: evidence from current financial crisis in OECD banks," Computational Economics, vol. 52, no. 1, pp. 275-297, 2018.

[6] H. T. Trinh, M. Kakinaka, D. Kim, and T. Y. Jung, "Capital structure and investment financing of small and mediumsized enterprises in Vietnam," Global Economic Review, vol. 46, no. 3, pp. 325-349, 2017.

[7] A. Salim, R. Amjesh, and C. S. S. Vinod, "SVM based lung cancer prediction using micro RNA expression profiling from NGS data," IEEE Transactions on Acoustics Speech \& Signal Processing, vol. 38, no. 4, pp. 599-609, 2016.

[8] L. Sun, H. Liu, L. Zhang, and J. Meng, "lncRScan-SVM: a tool for predicting long non-coding RNAs using support vector machine," PLoS One, vol. 10, no. 10, article 139654, 2015.

[9] Z. Ju, J. Z. Cao, and H. Gu, "Predicting lysine phosphoglycerylation with fuzzy SVM by incorporating k-spaced amino acid pairs into Chou's general PseAAC," Journal of Theoretical Biology, vol. 397, pp. 145-150, 2016.

[10] P. Xu, F. Davoine, H. Zha, and T. Denœux, "Evidential calibration of binary SVM classifiers," International Journal of Approximate Reasoning, vol. 72, no. C, pp. 55-70, 2016.

[11] X. L. Li, D. X. Liu, C. Jia, and X. Z. Chen, "Multi-model control of blast furnace burden surface based on fuzzy SVM," Neurocomputing, vol. 148, no. 148, pp. 209-215, 2015.

[12] Y. D. Zhang, Z. J. Yang, H. M. Lu et al., "Facial emotion recognition based on biorthogonal wavelet entropy, fuzzy support vector machine, and stratified cross validation," IEEE Access, vol. 4, no. 99, pp. 8375-8385, 2017.

[13] J. Hang, J. Zhang, and M. Cheng, "Application of multi-class fuzzy support vector machine classifier for fault diagnosis of wind turbine," Fuzzy Sets \& Systems, vol. 297, no. C, pp. 128-140, 2016.

[14] H. Xie, H. Huang, J. Wu, and L. Liu, "A comparative study of surface EMG classification by fuzzy relevance vector machine and fuzzy support vector machine," Physiological Measurement, vol. 36, no. 2, pp. 191-206, 2015.

[15] Z. P. Cai and Z. B. He, "Trading private range counting over big IoT data," in 2019 IEEE 39th International Conference on Distributed Computing Systems (ICDCS), Dallas, TX, USA, 2019.
[16] X. Zheng and Z. Cai, "Privacy-preserved data sharing towards multiple parties in industrial IoTs," IEEE Journal on Selected Areas in Communications (JSAC), vol. 38, no. 5, pp. 968-979, 2020.

[17] A. Abdiansah and R. Wardoyo, "Time complexity analysis of support vector machines (SVM) in LibSVM," International Journal of Computer Applications, vol. 128, no. 3, pp. 28-34, 2015.

[18] T. Jaya, J. Dheeba, and N. A. Singh, "Detection of hard exudates in colour fundus images using fuzzy support vector machine-based expert system," Journal of Digital Imaging, vol. 28, no. 6, pp. 761-768, 2015.

[19] Y. PoliszcWei, J. Watada, and W. Pedrycz, "Design of a qualitative classification model through fuzzy support vector machine with type-2 fuzzy expected regression classifier preset," Ieej Transactions on Electrical \& Electronic Engineering, vol. 11, no. 3, pp. 348-356, 2016.

[20] M. Hayat and M. Tahir, "PSOFuzzySVM-TMH: identification of transmembrane helix segments using ensemble feature space by incorporated fuzzy support vector machine," Molecular BioSystems, vol. 11, no. 8, pp. 2255-2262, 2015.

[21] Y. Sun, H. Song, A. J. Jara, and R. Bie, "Internet of things and big data analytics for smart and connected communities," IEEE Access, vol. 4, pp. 766-773, 2016.

[22] I. S. Thaseen and C. A. Kumar, "Intrusion detection model using fusion of chi-square feature selection and multi class SVM," Journal of King Saud University-Computer and Information Sciences, vol. 29, no. 4, article S1319157816300076, 2017.

[23] S. Zhu, J. Zhou, and C. Meng, "Study and application of fuzzy support vector machine based on gray correlation analysis to streamflow forecasting," Journal of Hydroelectric Engineering, vol. 34, no. 6, pp. 1-6, 2015. 